\title{
1 WILMS TUMORIGENESIS IN HUMAN KIDNEY ORGANOIDS
}

3 Verena Waehle ${ }^{1,2}$, Rosemarie Ungricht ${ }^{3}$, Philipp S. Hoppe ${ }^{3}$, Joerg Betschinger ${ }^{1, *}$

4

$5 \quad{ }^{1}$ Friedrich Miescher Institute for Biomedical Research, $\mathrm{CH}-4058$ Basel

$6 \quad{ }^{2}$ Faculty of Sciences, University of Basel, $\mathrm{CH}-4003$ Basel

$7 \quad{ }^{3}$ Novartis Institutes for BioMedical Research, Novartis Pharma AG, CH-4056 Basel

$8 \quad{ }^{*}$ lead contact: joerg.betschinger@fmi.ch

\section{SUMMARY}

11 The loss or failure of cell differentiation is a hallmark of cancer, yet whether perturbation of

12 differentiation is causal or consequential to malignant transformation is largely unclear.

13 Wilms tumor is the most widespread kidney cancer in children. Here, we establish a model

14 for Wilms tumorigenesis in human kidney organoids. We show that loss of the tumor

15 suppressor WT1 during organoid formation induces overgrowth of kidney progenitor cells at

16 the expense of differentiating tubules. Functional and gene expression analyses demonstrate

17 that absence of WT1 halts progenitor cell progression at a pre-epithelialized cell state and

18 recapitulates the transcriptional changes detected in a subgroup of Wilms tumor patients

19 with ectopic myogenesis. By "transplanting" WT1 mutant cells into wild-type kidney

20 organoids, we find that their propagation requires an untransformed microenvironment.

21 Genetic engineering of cancer lesions in human organoids therefore permits phenotypic 22 modeling of tumor initiation and progression, and complements the current toolbox of pre-

23 clinical Wilms tumor models. 


\section{INTRODUCTION}

2 Tumor initiation and progression is typically studied in genetically engineered mice (Kersten 3 et al, 2017), whose relevance to human disease is limited by species- and strain-specific mechanisms, and the artificial induction of multiple oncogenes at the same time. Most human cancer models, in contrast, employ patients' cells that are derived from tumor resections or biopsies, typically at a late stage of the disease, and that are grown as cell lines on tissue culture plastic in $2 D$, as tumor spheres in $3 D$, or as patient-derived xenografts (PDX) in mice (Hynds et al, 2018). Continuous growth and evolution of tumor cells in non-physiological environments imposes artificial selections (Ben-David et al, 2019) that can expose vulnerabilities irrelevant to the disease, which are thought to contribute to the poor clinical predictiveness of cancer models, in particular of tumor cell lines. To enhance clinical translatability, preclinical models are needed that more accurately represent the complexity of human cancers, such as tumor etiology, tumor heterogeneity and tumor environment.

Patient tumor-derived organoids preserve tumor heterogeneity, stages of tumor progression and drug responses (Clevers \& Tuveson, 2019). Conversely, engineering of cancer lesions into wild-type organoids induces growth factor-independent growth and predisposes to tumor formation upon xenotransplantation (Kawasaki et al, 2020; Fumagalli et al, 2017; Bian et al, 2018; Ogawa et al, 2018; Matano et al, 2015; Drost et al, 2015). Acquisition of disease traits is, in fact, evident in engineered organoids prior to transplantation: Introduction of colorectal cancer mutations into intestinal organoids, and of brain cancer lesions into induced pluripotent stem cell (iPSC)-derived cortical organoids were shown to promote transcriptional signatures that resemble premalignant adenoma (Matano et al, 2015), and glioblastoma and primitive neuroectodermal tumors (Ogawa et al, 2018; Bian et al, 2018), respectively. Similarly, addition of TGF $\beta$ to intestinal organoids derived from adenomas directs progression into a mesenchymal colorectal cancer phenotype (Fessler et al, 2016). Organoids may therefore be suitable to model tumor initiation and progression in vitro. Here, we test this possibility by introducing Wilms tumor patient lesions into iPSC-derived human kidney organoids.

Wilms tumor is the most common kidney cancer in childhood and accounts for about $7 \%$ of all pediatric cancers (Treger et al, 2019). Stalled nephrogenesis is thought to be the major cause of disease. This is supported by the transcriptional similarity of Wilms tumors with fetal 
1 cell types and by the function of several Wilms tumor oncogenes and tumor suppressors in normal kidney development. The diversity of genes that are mutated in Wilms tumor patients is larger than in other childhood tumors, and includes homozygous inactivation of the tumor suppressor Wilms tumor 1 (WT1), biallelic expression of insulin-like growth factor 2 (IGF2), neomorphic mutations in genes encoding the kidney transcription factors (TFs) SIX1 and SIX2, activation of the TF $\beta$-Catenin and disruption of miRNA biogenesis. The reason for this diversity is unclear. Some mutations, likely those that occur infrequently, may be associated with later stages of tumor development (Treger et al, 2019). However, gene expression analysis has suggested that Wilms tumorigenesis initiates in distinct cell types of origin and is influenced not only by which genes or pathways are mutated, but also by the developmental context in which genetic lesions occur (Gadd et al, 2012). Nephrogenesis may therefore be particularly vulnerable to transformation.

Here, we exploited human kidney organoids and showed that genetic knockout (KO) of WT1 induces overgrowth of nephron progenitor cells (NPCs) at the expense of tubular and glomerular differentiation. Further characterization revealed progression into an organoid state that transcriptionally and phenotypically resembles a subtype of Wilms tumor patients, as well as arrest of NPC differentiation at a pre-epithelialized cell state. Our study therefore establishes modeling of Wilms tumor initiation and progression in human kidney organoids, and defines transcriptional and phenotypic hallmarks of cellular transformation in the absence of WT1.

\section{RESULTS}

WT1 deletion inhibits NPC epithelialization and differentiation, and induces organoid hyperplasia

We generated kidney organoids using an adaptation of a two-step differentiation protocol (Morizane et al, 2015) (Ungricht et al., unpublished) that steers pluripotent iPSCs via intermediate mesoderm into SIX2-expressing NPCs within 9 days (d) in 2D, and NPCs into organoids within 12 days in 3D. Organoids contain podocytes expressing WT1, PODXL and NPHS1, distal tubules expressing the general epithelial marker EPCAM, and proximal tubules that are additionally labeled by LTL (Figure S1A-C). For KO of WT1 we infected iPSCs harboring 
1 a Doxycyline (DOX)-inducible Cas9 protein (Ungricht et al., unpublished) with lentiviruses 2 driving expression of a WT1-specific gRNA (gRNA1), a red fluorescent protein (RFP) and a 3 puromycin resistance gene, and selected for viral integration in iPSCs by treating with puromycin for $6 \mathrm{~d}$. Since the formation of Wilms tumor subtypes is thought to be influenced by the specific stage of kidney development in which mutations occur (Gadd et al, 2012), we induced genome editing by adding DOX at different stages of kidney organoid differentiation: in iPSCs prior to differentiation (KO ${ }^{\mathrm{iPSC}}$ ), during intermediate mesoderm specification (KO $\left.{ }^{\mathrm{d} 4-7}\right)$, during NPC differentiation (KO ${ }^{\mathrm{dg}-11}$ ) and during nephrogenesis (KO $\left.{ }^{\mathrm{d11}-14}\right)$. WT1 KO efficiency at the NPC stage, as determined by flow cytometry, was approximately $80 \%$ in $\mathrm{KO}^{\mathrm{iPSC}}$ and $70 \%$ in $\mathrm{KO}^{\mathrm{d} 4-7}$ when compared to uninduced cells (Figure S1D). WT1 depletion efficiencies at d21 were comparable for all deletion time-points (Figure $1 \mathrm{~A}$ ).

At d21, control and WT1 mutant organoids were of similar size, except for those derived from KO ${ }^{\text {iPSC }}$ cells, which were considerably smaller (Figure S1E). Culture until d30, however, revealed that $\mathrm{KO}^{\mathrm{iPSC}}$ organoids remained small, that $\mathrm{KO}^{\mathrm{d4-7}}$ and $\mathrm{KO}^{\mathrm{dg}-11}$ organoids overgrew, and that growth of $\mathrm{KO}^{\mathrm{d} 11-14}$ organoids was similar to controls. Profiling with cell type-specific

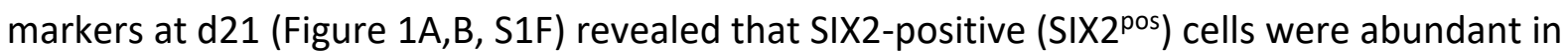
$\mathrm{KO}^{\mathrm{iPSC}}$ and $\mathrm{KO}^{\mathrm{d} 4-7}$ organoids ( $50 \%$ and $38 \%$ of all cells, respectively), but lower in $\mathrm{KO}^{\mathrm{d} 9-11}(17 \%)$ and absent from $\mathrm{KO}^{\mathrm{d} 11-14}$ organoids similar to controls. In addition, the proliferation marker $\mathrm{KI} 67$ was elevated, in particular in $\mathrm{KO}^{\mathrm{iPSC}}, \mathrm{KO}^{\mathrm{d} 4-7}$ and $\mathrm{KO}^{\mathrm{d} 9-11}$ organoids. Many KI67-positive $\left(\mathrm{KI} 67^{\text {pos }}\right)$ cells co-expressed SIX2. The specificity of this phenotype to depletion before d11 correlates with the downregulation of NPC genes and the induction of differentiation markers at this time-point (not shown), suggesting that overgrowth requires the deletion of WT1 in NPCs.

PODXL- and NPHS1-expressing podocytes were strongly reduced in all WT1 mutant organoids, including $\mathrm{KO}^{\mathrm{d11}-14}$ organoids (Figure 1B). Strikingly, the few detectable PODXL-positive cells co-expressed WT1, demonstrating that they are descendants of un-edited NPCs or NPCS harboring in-frame WT1 mutations. EPCAM ${ }^{\text {high }}$ and LTL-positive tubules were also reduced, indicating impaired formation of tubules (Figure $1 B, C$ ). The reduction of EPCAM ${ }^{\text {high }}$ cells was, however, less pronounced than the loss of podocytes. Defective nephrogenesis in $\mathrm{KO}^{\mathrm{d} 11-14}$ organoids in the absence of SIX2 deregulation suggests that WT1 drives exit from the NPC state, tubular differentiation and podocyte formation through independent mechanisms. We 
1 sought to validate these findings with two independent WT1 gRNAs and found a reduction of WT1 and EPCAM ${ }^{\text {high }}$, maintenance of SIX2, elevation of KI67, and co-expression of SIX2 and $\mathrm{KI} 67$ in $\mathrm{KO}^{\mathrm{iPSC}}$ organoids. Notably, phenotypic strength scaled with the KO efficiency of the respective gRNA, demonstrating on-target specificity of WT1 KO (Figure S1G,H).

While quantifying EPCAM expression, we found a significant expansion of EPCAM ${ }^{\text {mid }}$ cells in $\mathrm{KO}^{\mathrm{iPSC}}$ and $\mathrm{KO}^{\mathrm{d4}-7} \mathrm{~d} 21$ organoids (Figure $1 \mathrm{C}, \mathrm{S} 1 \mathrm{~F}, \mathrm{I}$ ). A significant proportion of these cells expressed SIX2 (33\% and 26\%, respectively). 9\% of EPCAM ${ }^{\text {mid }}$ cells in $\mathrm{KO}^{\mathrm{d} 9-11}$ organoids also co-expressed SIX2, although the total number of EPCAM ${ }^{\text {mid }}$ cells was not significantly increased. $\mathrm{KO}^{\mathrm{d11}-14}$ organoids, in contrast, were indistinguishable from controls. Immunofluorescence confirmed co-expression of EPCAM and SIX2 in cells that were arranged in epithelial-like sheets (Figure S1J), and at levels that were lower than in fully differentiated tubules. During organoid development EPCAM ${ }^{\text {mid }}$ cells emerged as early as $d 7$, preceding the formation of EPCAM ${ }^{\text {high }}$ cells (Figure $1 C$ ). These cells are therefore likely equivalent to EPCAM $^{\text {dim }}$ cells in the human fetal kidney (Pode-Shakked et al, 2017), which are kidney progenitor intermediates undergoing a mesenchymal-epithelial-transition (MET) and bridging the differentiation of EPCAM ${ }^{\text {low }}$ NPCs into EPCAM ${ }^{\text {high }}$ renal vesicle cells. WT1 is therefore required for progression beyond a pre-epithelial SIX2 ${ }^{\text {pos }} /$ EPCAM $^{\text {mid }}$ NPC transition state, but not for initiation of MET.

While the initial induction of EPCAM was unperturbed in mutant organoids (Figure 1C), we noted increased SIX2 protein levels in NPCs at d9 (Figure S1K). It is conceivable that this SIX2 de-repression is causal for progenitor overgrowth, and that a similar mechanism underlies Wilms tumor formation in patients with neomorphic SIX2 ${ }^{\mathrm{Q} 177 \mathrm{R}}$ mutations (Walz et al, 2015; Wegert et al, 2015). To test this possibility, we generated iPSCs with DOX-inducible mCherry::T2A::SIX2 and mCherry::T2A::SIX2 ${ }^{\mathrm{Q} 177 \mathrm{R}}$ transgenes. Induction of SIX2 by adding DOX from d7, but not d9 of organoid development onwards resulted in smaller organoids that, compared to uninduced controls, had fewer EPCAM ${ }^{\text {high }}$ - and LTL-positive tubules and WT1positive glomeruli (Figure S2A-D). In both, d7- and d9-induced organoids, however, KI67 expression was unchanged. SIX2, despite heterogeneous and mosaic expression, was detectable in differentiated EPCAM- and LTL-positive tubule cells at d21 (Figure S2D). SIX2 overactivation is therefore not sufficient to induce overproliferation or impair NPC epithelialization. 
1 Taken together these observations suggest that the removal of WT1 halts NPC progression at

2 a proliferating SIX2 $2^{\text {high }} /$ EPCAM $^{\text {mid }}$ transition state, which impairs the subsequent formation of

3 tubules and podocytes and, instead, leads to organoid hyperplasia. We found that overactivation of SIX2 was not sufficient to recapitulate these phenotypes, arguing for additional WT1 targets. Tubule and podocyte differentiation defects in $\mathrm{KO}^{\mathrm{d11}-14}$ organoids indicate roles of WT1 in nephrogenesis that are independent of SIX2 silencing and consistent with WT1 stabilizing an epithelial-mesenchymal hybrid state (Sampson et al, 2014) and activating podocyte-specific genes (Kann et al, 2015).

\section{WT1 drives developmental transcription}

11 We decided to define the transcriptional changes induced by absence of WT1, and performed RNAseq of $\mathrm{KO}^{\mathrm{iPSC}}$ and $\mathrm{KO}^{\mathrm{d} 4-7}$ cells and respective controls at different time-points during organoid development. Principle component (PC) analysis identified PC2 associated with mesoderm specification and PC1 with further organoid development (Figure S3A). Control and $\mathrm{KO}$ samples were indistinguishable up to $\mathrm{d} 12$, but segregated along PC1 at $\mathrm{d} 21$. Consistent with formation of podocytes and tubules in KO organoids (Figure 1B), KO ${ }^{\mathrm{iPSC}}$ and $\mathrm{KO}^{\mathrm{d4}-7} \mathrm{~d} 21$ samples did not overlap with d9 NPC, d11 or d12 samples.

k-means clustering of significantly changing transcripts revealed 17 gene clusters that are dynamically regulated during organoid development and/or dysregulated in WT1 mutants (Figure 2A,B, S3B; Table S1). These include clusters of genes that are induced or repressed during organoid development and unchanged in mutants (clusters 5, 7, 11 and 14), and clusters that are deregulated in specific cell states, in particular in d21 organoids (clusters 1,8 and 13). Notably, we did not identify clusters that are coherently mis-regulated in mutants, suggesting that WT1's target genes depend on the developmental context. Also, expression defects in $\mathrm{KO}^{\mathrm{iPSC}}$ and $\mathrm{KO}^{\mathrm{d} 4-7}$ organoids, despite distinct growth rates (Figure S1E), are very similar, and indistinguishable at d21.

Since WT1 KO perturbs the cellular composition of mutant organoids, we wondered if any of the gene clusters reflect cell type-specific transcription. We made use of published gene-sets that discriminate cell types of the human fetal kidney (Lindström et al, 2018) and calculated 
1 to be most similar to markers of relevant cell types in the developing kidney (Figure 2C).

2 Notably, cluster 11 was not changed, and cluster 17 only transiently deregulated in KO

3 organoids and compensated for at d21 (Figure S3B). We validated cell type-specificity of

4 clusters 1, 8 and 13 genes by visualizing their expression in t-distributed stochastic neighbor

5 embedding (t-SNE) maps of single cell RNAseq (scRNAseq) of the week 16 human fetal kidney

6 (Hochane et al, 2019). This confirmed co-expression with gene-sets of kidney progenitor cells

7 (M1), proliferating intermediates (M12 and M13), and podocytes (M6) and proximal tubule

8 cells (M9), respectively (Figure 2D).

9 Collectively, the transcriptional defects in developing WT1 mutant organoids correlate with

10 the phenotypic persistence of kidney progenitor cells (cluster 1 ) and reduced tubular

11 differentiation (cluster 13). Although we cannot exclude that upregulation of cluster 8 genes

12 reflects growth of a proliferating transit amplifying cell population, we note (1) that cluster 8

13 genes, similar to cell cycle-specific markers (Liu et al, 2017), are downregulated specifically at

$14 \mathrm{~d} 21$ of organoid development, suggesting cell type-independent transcription (Figure 2B,

$15 \mathrm{~S} 3 \mathrm{C}$ ), and (2) that a significant proportion of SIX2-positive cells co-express KI67 (Figure 1A), whose encoding gene MKi-67 is a cluster 8 gene (Table S1). Upregulation of cluster 8 genes is therefore likely due to NPC hyperproliferation rather than the persistence of an additional transit amplifying cell population.

\section{WT1 KO organoids recapitulate transcriptional changes in Wilms tumors}

Consistent with cell type-specific expression, gene ontology (GO) term analysis (Table S1) revealed enrichment of cell cycle and metabolic processes in clusters 8 and 13, respectively (Figure S3D). Surprisingly, cluster 1 - despite containing key NPC TFs such as SIX1, SIX2, EYA1 or MEOX1 - was highly enriched for genes with skeletal muscle function, such as the TFs MYOD1 and MYOG (Table S1). Notably, expression of muscle-specific genes defines a subclass of Wilms tumors (Miyagawa et al, 1998; Gadd et al, 2012, 2017). To test if gene expression changes recapitulate Wilms tumorigenesis, we compared alterations in d21 organoids with transcriptional changes in patient tumors. For this we profiled transcription in Wilms tumor patients and respective normal tissue (WT) (Gadd et al, 2017). To distinguish Wilms tumors from other kidney cancer types, we also included kidney chromophobe tumors (KICH) (Davis et al, 2014), kidney renal papillary cell carcinoma (KIRP) (Network et al, 2016) and kidney renal 
1 clear cell carcinoma (KIRC) (Creighton et al, 2013) into the analysis. Pairwise comparison

2 (Figure S3E) revealed that transcriptional defects in organoids correlated strongest with 3 alterations in WT, but less with $\mathrm{KICH}, \mathrm{KIRP}$ and KIRC patients. By focusing on gene clusters, we 4 found that both the magnitude and the directionality of cluster 1 and 8 deregulation in $\mathrm{d} 21$ 5 organoids was conserved specifically in WT patients (Figure 2B, 3A, S3B,F). Downregulation 6 of the differentiation-specific cluster 13, in contrast, was also observed in $\mathrm{KICH}$ patient 7 samples. Unsupervised clustering of cluster 1 genes in Wilms tumor samples (Figure 3B) 8 identified a group of genes, including SIX1, SIX2, EYA1 and MEOX1, that is strongly 9 upregulated in all patient samples, and two groups of genes containing myogenic TFs, such as MYOD1 and MYOG, and structural muscle genes, such as MYLPF and TNNC2, that are

11 dysregulated in only a subset of the patients.

12 We therefore conclude that transcriptional changes in WT1 mutant kidney organoids recapitulate defects in Wilms tumors, in particular the induction of NPC- and muscle-specific genes in cluster 1 and of cell-cycle-associated genes in cluster 8 , and the downregulation of differentiation-specific transcripts in cluster 13.

\section{Niche signals propagate WT1 mutant NPCs}

The persistence of SIX2 ${ }^{\text {pos }}$ cells and NPC transcription in WT1 KO organoids may result from a block or a delay in developmental progression. We reasoned that serial passaging in differentiation-promoting conditions would discriminate between the two by enforcing the commitment of delayed but not of blocked mutant cells. To do so, we aggregated single cells derived from $\mathrm{KO}^{\mathrm{d} 4-7} \mathrm{~d} 21$ organoids and exposed them to organoid-forming conditions for $12 \mathrm{~d}$ (corresponding to d9-d21 of iPSC differentiation), and repeated this procedure for up to four passages (Figure 4A). Since niche signals impact tumor growth and progression (Hanahan \& Coussens, 2012), we also tested the role of environment signaling in passaging of SIX2 ${ }^{\text {pos }}$ cells. We therefore added defined ratios (0\%, 10\%, 25\%, 75\% and 90\%) of GFP-expressing wild-type d9 NPCs to dissociated $\mathrm{KO}^{\mathrm{d4}-7}$ organoids during the aggregation step at the beginning of each passage, expecting that the wild-type kidney structures formed by these NPCs would provide niche signals to KO cells. Quantifying the percentage of GFP-expressing cells in chimeric organoids (Figure 4B) showed stable contribution of KO cells over passages, demonstrating that $\mathrm{KO}^{\mathrm{d} 4-7} \mathrm{~d} 21$ organoid cells grew as fast as wild-type $\mathrm{d} 9 \mathrm{NPCs}$. Un-edited control $\mathrm{d} 21$ 
1 organoid cells proliferated less (Figure S4A). The mutant cell type composition, in contrast,

2 varied across passages and mixing ratios (Figure 4B,C, S4C): In the absence (0\%) or presence 3 of $10 \%$ and $25 \%$ wild-type cells, the fraction of SIX $2^{\text {pos }}$ cells increased from approximately $30 \%$

4 to $60 \%$ after the first passage, but gradually declined during further passaging. Un-edited control cells, in contrast, did not gain SIX2 expression (Figure S4B). In the presence of $75 \%$ wild-type cells, the percentage of SIX $2^{\text {pos }}$ cells remained at $30 \%$ over passages for at least $60 \mathrm{~d}$. Presence of $90 \%$ wild-type cells stabilized SIX2-expression in $10 \%$ of $\mathrm{KO}^{\mathrm{d} 4-7}$ cells after the first passage. Therefore, paracrine signaling by wild-type cells, particularly at a wild-type:mutant cell ratio of 3:1, supports self-renewal of $\mathrm{KO}^{\mathrm{d} 4-7} \mathrm{SIX} 2^{\text {pos }}$ cells. At and below mixing ratios of 1:3, $\mathrm{SIX} 2^{\text {pos }}$ cells were lost during passaging. This was not accompanied by an induction of EPCAM $^{\text {high }}$ cells (Figure $4 \mathrm{~B}$ ) and therefore not due to overt tubular differentiation.

12 Passaging in the presence of wild-type NPCs is not comparable to growth in mature tissues, 13 such as during tumor progression or upon transplantation into PDX mouse models. We therefore decided to use ruptured GFP-expressing d21 organoids as a differentiated cell substrate for passaging of WT1 mutant cells (Figure 4A). We estimated that d21 organoids contain $150,000-200,000$ cells, to which we added 12,500 and 25,000 cells of RFP-expressing control or mutant single cells, corresponding to a mixing ratio of approximately $80-90 \%$. $\mathrm{KO}^{\mathrm{d} 4-7}$ d21 cells expanded visibly (Figure 4D), expressed SIX2 and KI67 and caused organoid overgrowth (Figure 4D, S4D,E). This was in contrast to un-edited d21 cells that contributed poorly to chimeric organoids. It is possible that this difference is due to different integration efficiencies of the two cell populations and not because of unrestricted growth of mutant cells. We therefore tested control d9 NPCs and found that they integrated equally well as $\mathrm{KO}^{\mathrm{d4}-7} \mathrm{~d} 21$ cells and contributed efficiently to EPCAM-positive epithelia. However, these cells did not induce organoid overgrowth or maintain SIX2 expression (Figure S4D-F). Passaging in d21 kidney organoids therefore enables persistence of SIX2 and proliferation of WT1 mutant cells. Notably this did not require extrinsic CHIR and FGF9 (Figure S4G-I), and is therefore independent of differentiation- and growth-promoting culture conditions.

We conclude that kidney organoids can be exploited for tumor cell transplantation and growth in a developmental (d9 NPCs) and mature (d21 organoids) tissue context. Our 
1 ectopic growth of mutant NPCs, specifically a developmental block induced by absence of WT1 and pro-self-renewal signals from a wild-type niche environment.

\section{DISCUSSION}

$5 \quad$ KO studies in mice have identified successive functions of WT1 during nephrogenesis, while WT1 mutations in humans predispose to familial forms of Wilms tumor and are found in 10-20\% of patients (Hastie, 2017; Treger et al, 2019). Our WT1-deletion analysis in human kidney organoids consolidates previous developmental and pathological observations:

WT1 is necessary for survival of the metanephric mesenchyme in mice (Kreidberg et al, 1993). KO of WT1 in human iPSCs, in contrast, did not prevent the differentiation into NPCS (Figure S1K). Although we can't exclude that this is because of species-specific functions of WT1 or because of non-cell autonomous rescue by unrecombined wild-type cells (Figure 1B, S1D), we propose that organoid culture conditions make up for WT1 deficiency: Deletion of FGF receptor 1 (Fgfr1) and Fgfr2, and of Fgf20 and Fgf9 in mice causes phenotypes that are reminiscent of loss of WT1 (Barak et al, 2012; Poladia et al, 2006). WT1 directly binds to and regulates the transcription of several Fgf genes, and treatment of WT1-mutant embryonic kidneys with recombinant FGF20 rescues cell death (Motamedi et al, 2014). We therefore speculate that extrinsic FGF9, added between d7 and d14 of organoid formation, suppresses the pro-apoptotic effect of WT1 loss, and exposes WT1's oncogenic role in developmentally more advanced NPCs.

In mice, WT1 drives the epithelialization of differentiating NPCs at least in part by promoting Wnt4 transcription (Essafi et al, 2011; Berry et al, 2015). In KO organoids, we found that a large fraction of ectopic SIX2 ${ }^{\text {pos }}$ cells expressed EPCAM ${ }^{\text {mid }}$ levels and that the transcriptional induction of WNT4 was impaired (Table S1), arguing that WT1 deficient human NPCs are developmentally arrested while undergoing MET (Pode-Shakked et al, 2017). This is reminiscent of the formation of heterogeneous tumors with few epithelial elements in human patients with WT1 mutations (Schumacher et al, 1997), although WT1 lesions have been reported in epithelial cells of Wilms tumor patient-derived organoids (Calandrini et al, 2020). Mutant NPCs may therefore occasionally exit the progenitor state and complete MET, which 
1 is consistent with the observation that epithelialization in mutant organoids was less 2 perturbed than glomerulogenesis (Figure 1A,C).

3 WT1 deletion in mice results in hyperplasia only in the context of increased IGF2 transcription 4 (Huang et al, 2016). Overgrowth of WT1 mutant organoids, in contrast, did not require the genetic perturbation of IGF2, likely because IGF2 protein was induced in the absence of WT1 (Figure 1B). We found that the magnitude of this induction correlated with the persistence of SIX2 ${ }^{\text {pos }}$ cells (Figure 1B), suggesting that the two phenotypes are linked. Surprisingly, IGF2 mRNA was not upregulated (Table S1), indicating that WT1 inhibits IGF2 expression through post-transcriptional mechanisms, such as via miRNAs (Bharathavikru et al, 2019).

Loss of WT1 in humans is thought to transform immature kidney progenitor cells (Treger et al, 2019). The timing of WT1 deletion, indeed, revealed that KO before $d 11 / 12$ of organoid development was required for overgrowth of SIX2 ${ }^{\text {pos }}$ cells (Figure $1 A, B$ ), matching with the terminal differentiation of endogenous NPCs. Similarly, expression of the NPC gene cluster 1 was maintained in $\mathrm{KO}^{\mathrm{iPSC}}$ and $\mathrm{KO}^{\mathrm{d} 4-7}$ organoids from $\mathrm{d} 9$ onwards (Figure $2 \mathrm{~A}, \mathrm{~B}$ ). We therefore conclude that ablation of WT1 impairs exit from the NPC state. Passaging in organoids showed that proliferating mutant SIX2 ${ }^{\text {pos }}$ cells can be maintained long-term, but revealed the importance of environmental signaling. In particular, we were not able to propagate SIX2 $2^{\text {pos }}$ cells in the absence of wild-type kidney cells. We note that primary Wilms tumor biopsies can be repeatedly passaged as spheroids (Wegert et al, 2020) and organoids (Calandrini et al, 2020 ) in 3D. Although growth conditions differ, it is worth considering the possibility that WT1 KO kidney organoids recapitulate an early stage of the disease and that further tumor progression leads to independence of paracrine signals.

Although the similarity of transcriptional changes in Wilms tumor patients and WT1 mutant kidney organoids was moderate (Figure S3E), we note that non-metanephric mesenchymederived cell types, such as collecting duct or vasculature, are absent from organoids (Morizane et al, 2015; Wu et al, 2018). Changes in organoids were more similar to Wilms tumors than other kidney cancers, and in particular to a subgroup of patients with upregulation of musclespecific transcripts. Loss of WT1 in Wilms tumor patients is associated with ectopic myogenesis (Miyagawa et al, 1998; Gadd et al, 2012), and in mouse NPCs with induction of a muscle gene expression signature (Berry et al, 2015). The origin of these muscle cells is unclear and may involve WT1 inhibiting mesodermal muscle differentiation (Miyagawa et al, 
1 1998; Berry et al, 2015). Skeletal muscle progenitor TFs, such as MEOX2 and PAX7 (Chal \& 2 Pourquié, 2017), were induced only late in d21 organoids and together with muscle TFs, such 3 as MYOG and MYOD1 (Table S1). This is inconsistent with co-development of muscle and nephrons, and suggests that muscle cells may result from the de-differentiation and ectopic re-differentiation (Shukrun et al, 2014) or the trans-differentiation of Wilms tumor cells.

6 Taken together, we here provide a framework for tracking tumor initiation and progression 7 in human iPSC-derived organoids. Our experimental approach is restricted to pediatric cancers with few genetic inducers, limited by availability of robust organoid protocols, and confined by inductive differentiation regimes that can mask non-cell autonomous phenotypes. Tumorigenesis modeling in organoids provides access to the cancer cell type of origin, to crosstalk between different oncogenes and tumor suppressors, to interactions with the niche environment, and, eventually, to organotypic and phenotypic platforms for drug discovery and development.

\section{ACKNOWLEDGMENTS}

Sebastien A Smallwood and team (FMI) for library preparation and sequencing, Panagiotis Papasaikas and Michael B Stadler (FMI) for help with computational analysis, Laurent Gelman (FMI) for advice and help with confocal microscopy, Melanie Rittirsch for technical assistance, Lapo Morelli (Novartis) for help with virus production and infection, Matthias Mueller (Novartis) for providing the WT29 iPSC line, and Helge Grosshans, Susan Gasser (FMI) and Soeren Lienkamp (University of Zurich) for comments on the manuscript. V.W. acknowledges support by a Boehringer Ingelheim Fonds PhD fellowship, and J.B. from the Novartis Research Foundation.

\section{AUTHOR CONTRIBUTIONS}

VW, RU, PSH and JB conceived the study. VW designed (with input from RU) and performed all experiments and analyzed the data. RU adapted the organoid protocol and generated the WT29-iCas9 and GFP-expressing WT29-iCas9 lines. JB performed computational analyses. JB and PSH supervised the study. VW and JB wrote the manuscript with input from all authors. 


\section{COMPETING INTERESTS}

2 The authors declare no conflicts of interest.

\section{FIGURE LEGENDS}

$5 \quad$ Figure 1: Loss of WT1 arrests NPC progression and induces NPC overgrowth.

6 A: Quantification of subpopulations in pooled $\mathrm{d} 21$ un-edited, $\mathrm{KO}^{\mathrm{iPSC}}, \mathrm{KO}^{\mathrm{d4}-7}, \mathrm{KO}^{\mathrm{dg}-11}$ and $7 \mathrm{KO}^{\mathrm{d} 11-14}$ organoids by flow cytometry of indicated markers. Data is presented as mean $\%$ of 8 positive cells $+/$ - SD derived from $n=5$ (un-edited, $K \mathrm{O}^{\mathrm{iPSC}}, \mathrm{KO}^{\mathrm{d4-7}}$ ) or $\mathrm{n}=2\left(\mathrm{KO}^{\mathrm{dg}-11}, \mathrm{KO}^{\mathrm{d} 11-14}\right.$ ) 9 independent experiments. Two-sided student's t-test; $p$-value: $n s>0.05 ;{ }^{*} \leq 0.05 ; * * \leq 0.01$; $10 * * * \leq 0.001 ; * * * * \leq 0.0001 ;$ SIX2-Ki-67: asterisks correspond to the SIX2-KI67 population.

11 B: IF staining for the indicated markers in representative un-edited, $\mathrm{KO}^{\mathrm{iPSC}}, \mathrm{KO}^{\mathrm{d4} 4-7}, \mathrm{KO}^{\mathrm{d9}-11}$ and $12 \mathrm{KO}^{\mathrm{d} 11-14} \mathrm{~d} 21$ organoids. Scale bar: $100 \mu \mathrm{m}$. White boxed regions are shown in Figure S1J.

13 C: Flow cytometry-based quantification of EpCAM ${ }^{\text {high }}$, EPCAM $^{\text {mid }}$ and EpCAM ${ }^{\text {low }}$ populations 14 (see gating shown in Figure S1I) in pooled organoids of indicated time-points and genotypes. 15 Data is shown as mean \% of positive cells $+/-$ SD derived from $n=3$ (Day 7); $n=4$ (Day 9); $n=2$ 16 (Day 10); $\mathrm{n}=5$ (un-edited, $\mathrm{KO}^{\mathrm{iPSC}}, \mathrm{KO}^{\mathrm{d} 4-7}$; Day 21) or $\mathrm{n}=2$ (KO ${ }^{\mathrm{d} 9-11}, \mathrm{KO}^{\mathrm{d} 11-14}$; Day 21) independent 17 differentiation experiments. Two-sided student's t-test; $p$-value: $\mathrm{ns}>0.05 ;{ }^{*} \leq 0.05 ;{ }^{*} \leq 0.01$; asterisks are placed above the respective populations (EPCAM ${ }^{\text {high }}$ and EPCAM ${ }^{\text {mid }}$ ).

Figure 2: Absence of WT1 impairs developmental transcription.

21 A: k-means clustering of 7,626 genes significantly changing in any of the shown contrasts. Left: time course of wild-type organoid development; expression log2 fold changes (Log2FC) relative to iPSCs. Right: Changes in $\mathrm{KO}^{\mathrm{iPSC}}$ or $\mathrm{KO}^{\mathrm{d} 4-7}$ cells and at indicated time-points; Log2FC relative to un-edited control at each time-point.

B: Quantification of mRNA Log2FC of indicated gene clusters and in contrasts as specified in A.

C: Gene set overlap significance scores (see Methods for details) of 17 gene clusters with fetal kidney cell type gene-sets as defined in (Lindström et al, 2018). Z-scores are color-coded. diff = differentiate. 
1 D: t-SNE maps of scRNAseq data from week 16 human fetal kidney (Hochane et al, 2019).

2 Expression levels of indicated clusters and gene-sets (see $\mathbf{C}$ ) are color-coded. M12 Kidney prol.

$3 \mathrm{I}=$ M12 Kidney proliferation I; M13 Kidney prol. II = M13 Kidney proliferation II; Prox = 4 proximal.

6 Figure 3: WT1 KO organoids recapitulate the transcriptional changes of a Wilms tumor 7 patient subgroup.

8 A: Log2 FCs of indicated gene clusters in WT, KICH, KIRP and KIRC patient samples relative to 9 the corresponding normal tissue.

10 B: Unsupervised clustering of Log2FC of cluster 1 genes in WT relative to corresponding normal tissue. Clusters containing nephron progenitor genes or muscle genes are highlighted.

\section{Figure 4: Non cell-autonomous regulation of self-renewal and heterogeneity.}

14 A: Schematic of the experimental flow shown in A-C (upper) and D (lower).

B: Quantification of indicated cell populations in chimeric organoids after passaging with indicated ratios of wild-type GFP expressing d9 NPCs and at indicated passages. p0 indicates $\mathrm{KO}^{\mathrm{d4}-7} \mathrm{~d} 21$ organoids that were used as starting material for passaging. Data is shown as mean \%+/-SD from $n=1$ or 2 independent experiments. (\% of all cells) is relative to all cells of chimeric organoids, and (\% of $\mathrm{KO}^{\mathrm{d} 4-7}$ cells) is relative to all mutant cells, therefore excluding wild-type host cells.

C: IF staining at p3 of indicated markers and mixing ratios as outlined in A. Scale bar: $100 \mu \mathrm{m}$.

D: Images of organoids obtained after adding 12,500 RFP-positive un-edited d21 organoids

23 (top) and $\mathrm{KO}^{\mathrm{d4-7}} \mathrm{d} 21$ organoids (bottom) to ruptured wild-type GFP organoids. Images were recorded at indicated time-points using an Incucyte ${ }^{\circledR}$ system. Scale bar $1 \mathrm{~mm}$. 


\section{SUPPLEMENTAL INFORMATION}

\section{Figure S1: Related to Figure 1}

3 A: Overview of the kidney organoid protocol adapted from (Morizane et al, 2015); Prox = 4 proximal; Dist $=$ distal.

5 B: IF staining of the indicated markers in representative d14, d18 and d21 organoids.

6 C: Pearson correlation coefficients of pairwise comparisons between Log2FCs of indicated 7 organoid samples relative to corresponding iPSC samples. Expression data for d26, Morizane 8 and d26, Takasato are from (Wu et al, 2018).

9 D,G: Quantification of WT1-expressing cells in d9 NPCs with indicated genotypes: D un-edited, $10 \mathrm{KO}^{\mathrm{iPSC}}$ or KO $\mathrm{K}^{\mathrm{d} 4-7}$ cells, and G KO ${ }^{\mathrm{iPSC}}$ generated by indicated WT1 gRNAs. Data presented as, D 11 mean $\%$ of positive cells $+/-$ SD from $n=7$ independent experiments, and $\mathbf{G}$ from one 12 experiment.

13 E: Growth of organoids derived from un-edited, $\mathrm{KO}^{\mathrm{iPSC}}, \mathrm{KO}^{\mathrm{d4}-7}$, KO $\mathrm{KO}^{\mathrm{d}-11}$ and $\mathrm{KO}^{\mathrm{d} 11-14}$ cells 14 between $\mathrm{d} 11$ and $\mathrm{d} 30$. Areas were derived from Incucyte ${ }^{\circledR}$ images and are presented as mean $+/$ - SD for a minimum of $n=10$ organoids across all time-points.

$16 \mathrm{~F}, \mathrm{H}$ : Quantification of subpopulations expressing indicated markers in pooled $\mathrm{d} 21$ organoids

17 of indicated genotypes: $\mathbf{F}$ un-edited, $\mathrm{KO}^{\mathrm{iPSC}}, \mathrm{KO}^{\mathrm{d4}-7}, \mathrm{KO}^{\mathrm{d} 9-11}$ and $\mathrm{KO}^{\mathrm{d} 11-14}$, and $\mathbf{H} \mathrm{KO}^{\mathrm{iPSC}}$ generated by indicated WT1 gRNAs. Data is presented as, $\mathbf{F}$ mean $\%$ of positive cells +/- SD derived from $\mathrm{n}=5$ (un-edited, $\mathrm{KO}^{\mathrm{iPSC}}, \mathrm{KO}^{\mathrm{d4}-7} ; \mathrm{KI} 67$ and SIX2), $\mathrm{n}=2$ (KO ${ }^{\mathrm{d9}-11}, \mathrm{KO}^{\mathrm{d} 11-14} ; \mathrm{KI} 67$ and SIX2), $\mathrm{n}=5$ (un-edited, KO $\left.{ }^{\mathrm{iPSC}}, \mathrm{KO}^{\mathrm{d} 4-7} ; \mathrm{SIX2-EPCAM}\right)$ and $\mathrm{n}=1$ (KO ${ }^{\mathrm{d} 9-11}, \mathrm{KO}^{\mathrm{d11}-14} ;$ SIX2-EPCAM) independent experiments, and $\mathbf{H}$ one experiment. Note that the SIX2-EPCAM quantification includes data presented in the Day 21 panel of Figure 1C. Two-sided student's t-test; $p$-value: $\mathrm{ns}>0.05 ; * \leq 0.05 ; * * \leq 0.01 ; * * * \leq 0.001 ; * * * \leq 0.0001$.

I: Representative flow cytometry dot plots showing EPCAM and SIX2 staining in pooled d21 organoids as in E. Pink boxes and associated numbers indicate \% of cells that are EPCAM ${ }^{\text {high }}$, EPCAM $^{\text {mid }}$ and EPCAM $^{\text {low }}$. Blue boxes and associated numbers indicate $\%$ of cells that are SIX2 ${ }^{\text {pos }} /$ EPCAM $^{\text {high }}$, SIX2 $^{\text {pos }} /$ EPCAM $^{\text {mid }}$ or SIX2 $2^{\text {pos }} /$ EPCAM $^{\text {low }}$.

J: Magnification corresponding to boxes in Figure 1B. Images are overexposed to visualize EPCAM staining surrounding SIX2-positive cells (white arrowheads). Scale bar: $500 \mu \mathrm{m}$. 
$1 \quad$ K: SIX2 expression in d9 NPCs by flow-cytometry. The same conditions as in A are shown.

\section{$3 \quad$ Figure S2: Related to Figure 1.}

4 A: QPCR analysis of SIX2 transcription in d9 NPCs derived from the indicated lines that were untreated (no DOX) or treated with DOX starting from the indicated time-points. Data is presented as Log2FC relative to the untreated control. Data is shown as mean +/- SD derived

7 from $n=3$ differentiation experiments.

8 B: mCherry expression in pooled d21 organoids derived the indicated lines that were untreated (no DOX) or treated with DOX starting from the indicated time-points.

10 C: Quantification of subpopulations expressing indicated markers in pooled d21 organoids 11 derived from the indicated lines that were untreated (no DOX) or treated with DOX as in B.

12 Data is shown as \% of positive cells for one representative experiment.

13 D: IF staining of indicated markers in representative $\mathrm{d} 21$ organoids that were untreated (no 14 DOX) or treated with DOX as in B. mCherry represents induction of SIX2 or SIX2 ${ }^{\mathrm{Q} 177 R}$. White arrowheads indicate expression of SIX2 in LTL ${ }^{\text {pos }}$ tubules. Scale bar: $100 \mu \mathrm{m}$.

$17 \quad$ Figure S3: Related to Figures 2 and 3.

18 A: PCA of un-edited (un-ed.) $\mathrm{KO}^{\mathrm{iPSC}}$ and $\mathrm{KO}^{\mathrm{d4-7}}$ samples at the indicated time-points.

19 B,C: Quantification of mRNA Log2FC of, B the indicated gene clusters, and C of cell cycle genes, 20 and in contrasts as specified in Figure $2 A$. The cell cycle gene-set include $G_{1} S-$ and $G_{2} M-s p e c i f i c$ 21 gene-sets (Liu et al, 2017).

D: The top six GO terms that are enriched in each of the indicated gene clusters is shown. dev. $=$ development $;$ proc.$=$ process $;$ metab. $=$ metabolism; catab. $=$ catabolism .

E: Pearson correlation coefficients of pairwise comparisons between mean Log2FCs in KO $\mathrm{K}^{\mathrm{PPC}}$ and $\mathrm{KO}^{\mathrm{d} 4-7} \mathrm{~d} 21$ organoids relative to un-edited $\mathrm{d} 21$ organoids (d21 organoid), and kidney cancer patient samples relative to corresponding normal tissue. The 7'626 transcripts shown in Figure $2 \mathrm{~A}$ were used. $\mathrm{WT}=$ Wilms tumor; $\mathrm{KICH}=$ kidney chromophobe carcinoma; $\mathrm{KIRP}=$ kidney papillary carcinoma; KIRC = kidney clear cell carcinoma. 
1 F: Log2FCs of indicated clusters in WT, KICH, KIRP and KIRC patient samples relative to corresponding normal tissue.

Figure S4: Related to Figure 4.

5 A,B: Quantification of, A all, and B SIX2 ${ }^{\text {pos }}$ un-edited and $\mathrm{KO}^{\mathrm{d} 4-7}$ cells in chimeric organoids 6 after passaging in the presence of wild-type GFP-expressing d9 NPCs at indicated ratios after 7 passage 1. $\mathrm{p} 0$ indicates $\mathrm{KO}^{\mathrm{d} 4-7} \mathrm{~d} 21$ organoids that were used as starting material for passaging.

8 Data is shown as mean $\%+/$-SD for $n=2$ independent experiments. A: Percentages are relative 9 to all cells of chimeric organoids (\% of all cells). B: Percentages are relative to all mutant cells 10 (\% of $\mathrm{KO}^{\mathrm{d} 4-7}$ cells), and relative to all un-edited control cells (\% of un-edited cells). In both 11 cases, the GFP-expressing cells are excluded from the analysis.

$12 \mathbf{C}, \mathbf{D}, \mathbf{I}$ : IF staining of the indicated markers, $\mathbf{C}$ at passage 3 and indicated mixing ratios, $\mathbf{D} 15 \mathrm{~d}$ 13 after adding 12,500 RFP-expressing cells from un-edited d21 organoids (un-ed. d21, top), un14 edited d9 NPCs (un-ed. NPCs, middle), or $\mathrm{KO}^{\mathrm{d} 4-7} \mathrm{~d} 21$ organoids (KO ${ }^{\mathrm{d} 4-7} \mathrm{~d} 21$, bottom) to ruptured wild-type GFP-expressing organoids in the presence of FGF9/CHIR, and I after adding 12,500 RFP-expressing cells from $\mathrm{KO}^{\mathrm{d} 4-7} \mathrm{~d} 21$ organoids to ruptured wild-type GFP-expressing organoids in the absence of growth factors. Scale bar: $100 \mu \mathrm{m}$.

E,H: Growth of organoids, E after adding 12,500 (12.5k) or 25,000 (25k) RFP-expressing cells in the presence of FGF9/CHIR as specified in $\mathbf{D}$, and $\mathbf{H}$ after adding the indicated numbers of RFP-expressing $\mathrm{KO}^{\mathrm{d} 4-7} \mathrm{~d} 21$ organoid cells to ruptured wild-type GFP organoids and in the absence of growth factors. Areas were calculated from Incucyte ${ }^{\circledR}$ images and are presented as mean $+/$ - standard error of the mean (SEM) for $n=8$ organoids per condition.

F,G: Images of organoids obtained after adding, F 12,500 RFP-expressing un-edited d9 NPCS to ruptured wild-type GFP organoids in the presence of FGF9/CHIR, and G 12,500 RFPexpressing cells from $\mathrm{KO}^{\mathrm{d4-7}} \mathrm{d} 21$ organoids to ruptured wild-type GFP organoids in the absence of growth factors. Images were recorded at the indicated time-points using an Incucyte ${ }^{\circledR}$ system. Scale bar $1 \mathrm{~mm}$. 


\section{MATERIALS AND METHODS}

\section{Human iPSC culture}

3 WT29 iPSCs and their derivatives were cultured on Laminin (Biolaminin 521 LN; Biolamina \#LN521) in mTeSR1 (Stem Cell Technologies; \# 85850) plus 1\% Penicillin-Streptomycin (Thermo Fisher \#15140122). For passaging, cells were detached using TrypLE Express (Thermo Fisher \#12604013), and single-cell suspensions were re-plated in mTeSR1 supplemented with $2 \mu \mathrm{M}$ ROCK inhibitor (Y-27632 Dihydrochloride Tocris \#1254). Transgenic WT29 iPSCs were generated by transfecting PiggyBac expression vector and pBase (Villegas et al, 2019) using Lipofectamin Stem $^{\mathrm{TM}}$ (Thermo Fisher \#STEM00015) in OptiMem Reduced Serum Medium (Thermo Fisher \#319850629), and selected for stable integration in the presence of $100 \mu \mathrm{g} / \mathrm{ml}$ G418 (Thermo Fisher \#10131027). Inducible cells were further selected by exposing to $1 \mathrm{ug} / \mathrm{ml}$ Doxycycline (DOX; Clonetech \#631311) for $48 \mathrm{~h}$ and purifying the $30 \%$ of cells with mCherry expression closest to the median of the population.

\section{Kidney organoids}

We employed a two-step protocol, consisting of an adherent (d0-d9) part forming NPCs and a suspension (d9-d21) part generating organoids, that was adapted from (Morizane et al, 2015) (R. Ungricht, unpublished). Induction of SIX2 and SIX2 ${ }^{Q 177 R}$, and of Cas9 was achieved by treating with $1 \mu \mathrm{g} / \mathrm{ml}$ and $0.2 \mu \mathrm{g} / \mathrm{ml}$ Dox for indicated time-points, respectively. Adherent differentiation (d0-d9): 50'000-60'000 hiPSCs $/ \mathrm{cm}^{2}$ were plated in $\mathrm{mTeSR} 1$ supplemented with $2 \mu \mathrm{M}$ ROCK inhibitor into Laminin-coated 6-well plates. After at least $6 \mathrm{~h}$, medium was removed, cells gently washed with Dulbecco's Phosphate Buffered Saline (PBS; without magnesium and calcium; Thermo Fisher \#14190169), and differentiation induced by adding basic differentiation medium (BDM; advanced RPMI 1640 (Thermo Fisher \#12633012), 1\% Glutamax (Thermo Fisher \#35050038), 1\% Penicillin-Streptomycin) supplemented with $8 \mu \mathrm{M}$ CHIR99021 (Tocris \#4423) and $5 \mathrm{ng} / \mathrm{ml}$ Noggin (Peprotech \#120-10C) (= d0). Medium was changed after 2 days. At d4, cells were visually inspected for the presence of contracting colonies with bright halo-like outlines, followed by medium exchange to BDM containing $10 \mathrm{ng} / \mathrm{ml}$ Activin A (R\&D Systems \#338_AC). At d7, medium was changed to BDM supplemented with $10 \mathrm{ng} / \mathrm{ml} \mathrm{FGF9} \mathrm{(R \& D} \mathrm{Systems} \mathrm{\# 273-F9).} \mathrm{At} \mathrm{d9,} \mathrm{corresponding} \mathrm{to} \mathrm{the} \mathrm{NPC}$ 
1 state, cells were washed, dissociated from the cell culture dish using TrypLE and counted using

2 a Vi-CELL ${ }^{\mathrm{TM}}$ XR Cell Viability Analyzer (Beckmann).

3 Organoid differentiation in suspension culture (d9 onwards): $25^{\prime} 000$ or $50^{\prime} 000$ cells were seeded in $150 \mu$ l of BDM containing $3 \mu \mathrm{M}$ CHIR99021, 10 ng/ml FGF9 and $2 \mu \mathrm{M}$ ROCK inhibitor into Corning ${ }^{\circledR}$ Costar $^{\circledR}$ Ultra-Low Attachment 96-well round bottom plates (Sigma \#CLS700724EA). Surplus NPCs were frozen in CryoStor ${ }^{\circledR}$ CS10 (Stem Cell Technologies \#7930). To induce aggregation, the plate was briefly centrifuged at $90 \mathrm{~g}$ for 3 minutes (min). At $\mathrm{d} 10$ (d1 in suspension), $100 \mu \mathrm{l}$ of medium was replaced with $150 \mu \mathrm{l}$ of BDM plus $10 \mathrm{ng} / \mathrm{ml}$ FGF9. At d11 (d2), $100 \mu$ l of medium was replaced with $100 \mu$ l of BDM plus $10 \mathrm{ng} / \mathrm{ml} \mathrm{FGF9.} \mathrm{At} \mathrm{d14} \mathrm{(d5),} \mathrm{d16}$ (d7) and d18 (d9), $100 \mu$ l of medium was replaced with $100 \mu$ l of BDM without growth factors. During culture beyond $d 21$, medium was changed 3 times per week. Organoid growth was determined using an Incucyte ${ }^{\circledR}$ system: Brightfield images were recorded every $24 \mathrm{~h}$, or as indicated, and organoid sizes were calculated. Object recognition parameters were manually defined for each experiment, and the detected objects validated.

To generate single cell suspensions for flow cytometry or passaging, organoids were transferred into tubes using a cut P-1000 tip, and washed twice with PBS. A 1:1 mix of nonenzymatic cell dissociation solution (Thermo Fisher \#13151014) and 0.25\% Trypsin-EDTA (Thermo Fisher \#25200056) was added for 10 mins at $37^{\circ} \mathrm{C}$, and organoids were dissociated by pipetting up and down ten times. Trypsin was inactivated by adding $10 \%$ fetal calf serum (FCS; Bioconcept \#2-01F36-I) in PBS, washed with 1\% FCS and passed through a $50 \mu \mathrm{m}$ filter (BD Biosciences \#340632).

\section{Chimeric organoids}

For mixing with NPCs (Figure 4B,C, S4A-C), single cell suspensions of RFP-expressing un-edited or $\mathrm{KO}^{\mathrm{d} 4-7} \mathrm{~d} 21$ organoids were generated as described above, aggregated with freshly thawed GFP-expressing WT29-iCas9 d9 NPCs at indicated ratios to a total of 50,000 cells, and plated into Ultra-Low Attachment 96-well round bottom plates in $150 \mu \mathrm{I} \mathrm{BDM,} \mathrm{supplemented} \mathrm{with} 3$ $\mu \mathrm{M}$ CHIR99021, $10 \mathrm{ng} / \mathrm{ml}$ FGF9 and $2 \mu \mathrm{M}$ ROCK inhibitor. Culture was continued as detailed in "Organoid differentiation in suspension culture» above. At d12 of suspension culture, organoids were dissociated into single cell suspensions that were used for flow cytometry analysis and for passaging by aggregating with freshly thawed GFP-expressing WT29-iCas9 d9 
$1 \quad$ NPCs at respective ratios and subjecting to suspension culture. This was repeated for up to 2 four passages. After each passage, a minimum of six organoids was processed for 3 cryosectioning and immunofluorescence staining, as described below.

4 For mixing into d21 organoids (Figure 4D, S4E-I), GFP-expressing WT29-iCas9 d21 were 5 mechanically ruptured by pipetting them up and down five times in a P-200 tip. RFP6 expressing un-edited and $\mathrm{KO}^{\mathrm{d} 4-7} \mathrm{~d} 21$ organoids were dissociated into single cell suspensions 7 as described above, and un-edited RFP-expressing d9 NPCs were freshly thawed. After cell 8 counting, indicated cell numbers were added to ruptured organoids, plates briefly spun to 9 induce aggregation, and culture in BDM resumed as indicated, either in the presence or absence of $3 \mu \mathrm{M}$ CHIR99021 and 10ng/ml FGF9.

Molecular biology

13 The coding sequence of SIX2 was amplified from human iPSC CDNA, T2A sequences and 14 Gateway cloning sites added by polymerase chain reaction (PCR), and recombined into pDONR221 using Gateway technology (Thermo Fisher \#11789020 and \#11791020). The Q177R point mutation in SIX2 was introduced by PCR. Expression vectors were generated by recombining with a PiggyBac pPB-TRE-mCherry-DEST-rTA-HSV-neo expression destination vector. WT1 gRNA-encoding vectors are derived from pRSI16-U6-sh-UbiC-TagRFP-2A-Puro

19 (Cellecta \#SVSHU616-L).

Oligonucleotide sequences:

\begin{tabular}{|l|l|l|}
\hline SIX2 (gateway) & attB1-fwd & ggggacaagtttgtacaaaaaagcaggcttcaccATGTCCATGCTGCCCACCTTCG \\
\hline & attB2-rev & ggggaccactttgtacaagaaagctgggtcCTAGGAGCCCAGGTCCACGAGG \\
\hline mutagenesis SIX2 ${ }^{\text {Q177R }}$ & fwd & ttcaagaaccggcggcGgcgcgaccgggcggcc \\
\hline & rev & ggccgcccggtcgcgcCgccgccggttcttgaa \\
\hline T2A sequence & fwd & GAGGGCAGAGGAAGTCTCCTAACATGCGGTGACGTGGAGGAGAATCCTGGCCCA \\
\hline
\end{tabular}

$22 \mathrm{fwd}=$ forward sequence; rev = reverse sequence

\begin{tabular}{|l|l|l|}
\hline WT1 & $\# 1$ & TGTGTTTGCAGCCACAGCAC \\
\hline WT1 & $\# 2$ & GGTGTGGCAGCCATAGACCG \\
\hline WT1 & $\# 3$ & GCTGCCGGTGCAGCTGTCGG \\
\hline
\end{tabular}


2 Lentiviruses were produced in HEK293T cells. Prior to transfection, HEK293T cells were

3 seeded onto collagen I-coated 6-well tissue culture plates (BD biosciences \#346400) in packaging medium (DMEM (Thermo Fisher \#11965), supplemented with 10\% FCS and 1\% Non-essential amino acids (Thermo Fisher \#11140050)). The next day, cells were transfected with WT1 gRNA-encoding vectors and Cellecta packaging mix (Cellecta \#CPC-K2A) using the TransIT ${ }^{\mathrm{TM}} 293$ transfection reagent (Mirus Bio \#MIR 2700) in OptiMem Reduced Serum Medium (Thermo Fisher \#31985062). The next day, medium was changed to $1 \mathrm{ml}$ of packaging medium. After 3 days (d), the virus-containing supernatant was collected, filtered through a $50 \mu \mathrm{m}$ filter and stored at $-80^{\circ} \mathrm{C}$.

11 For virus titration, WT29-iCas9 cells were seeded into 6 -well plates $(200,000$ cells / well) in 12 mTeSR1 medium supplemented with ROCK inhibitor. After $7 \mathrm{~h}$, different volumes of viral supernatant were added to the cells. After $3 \mathrm{~d}$ with daily mTeSR1 medium changes, cells were detached and RFP fluorescence was measured by flow cytometry. Based on this titration, WT29-iCas 9 cells were transduced at a multiplicity of infection of 0.5 , and infected cells were selected with puromycin (Thermo Fisher \#A11138-03) for 6 days.

RNA isolation, cDNA synthesis, qPCR

RNA was isolated using RNeasy Mini Kits (Qiagen \#74104) and RNase-Free-DNase Sets (Qiagen \#79256) according to the manufacturer's instructions, and concentration determined using a NanoDrop (Thermo Fisher). cDNA was generated from at least $400 \mathrm{ng}$ of total RNA using SuperScript III Reverse Transcriptase (Thermo Fisher \# 18080044) using oligodT priming. 25 ng of cDNA was subjected to quantitative PCR (qPCR) on a Step One Plus ${ }^{\mathrm{TM}}$ Real-Time PCR System (Thermo Fisher) using the TaqMan Fast Universal PCR Master Mix (Thermo Fisher \# 4364103). Expression of SIX2 was quantified in technical duplicates using Universal Probe Library (UPL, Roche) Probe 88 together with custom-designed primer pairs: Fwd: ggcaagtcggtgttaggc, Rev: ggctggatgatgagtggtct, and multiplexing with a GAPDH probe (Thermo Fisher \# 4326317E) . 
2 Single live cell suspensions were washed with 1\% FCS in PBS and resuspended in flow 3 cytometry buffer (2\% FCS and $1 \mathrm{mM}$ ethylenediaminetetraacetic acid (EDTA; Thermo Fisher 4 \#AM9260G) in PBS).

5 For flow cytometry analysis of stained cells, dissociated cells were fixed using the BD 6 Cytofix/Cytoperm Fixation/Permeabilization Kit (BD Biosciences \#554714) according to the 7 manufacturer's instructions with the exception that $0.4 \%$ bovine albumin fraction $\mathrm{V}$ solution 8 (BSA, 7.5\%, Thermo Fisher \#15260037) in PBS was added to fixed cells before centrifugation. 9 Cells were incubated with primary antibodies in permeabilization buffer at $4^{\circ} \mathrm{C}$ for 60 mins, washed three times, incubated with secondary antibodies at $4^{\circ} \mathrm{C}$ for 60 mins, washed three

11 times and resuspended in permeabilization buffer for flow analysis. Antibodies were EPCAM12 Alexa Fluor (AF) 647 (Abcam \#ab239273, 1:200); Ki-67-FITC (eBisoscience \#11-5698-82, 13 1:200); SIX2 (Proteintech \#11562-1-AP, 1:100); WT1 (Abcam \#ab89901, 1:200); donkey-anti14 rabbit AF488 (Thermo Fisher \#A-21206, 1:500); donkey-anti-rabbit AF 594 (Thermo Fisher \#A21207, 1:500); donkey-anti-rabbit AF 647 (Thermo Fisher \#A-31573, 1:500). Analytic flow cytometry was performed on a BD LSRFortessa ${ }^{\mathrm{TM}}$ or BD LRSII ${ }^{\mathrm{TM}}$ device, cell sorting on a BD FACSAria ${ }^{\text {TM }}$ Fusion Cell Sorter. Data was analyzed in Flow Jo.

RFP-expression in fixed WT1 KO cells of chimeric organoids (Figure 4B, S4A,B) by flow cytometry was ambiguous. We therefore quantified the fraction of mutant cells by determining the percentage of GFP-expressing wild-type WT29-iCas9 cells and correcting for heterogeneous GFP expression.

Immunofluorescence

Kidney organoids were pooled, washed twice with PBS, and fixed with $4 \%$ paraformaldehyde (PFA) in PBS for 20 mins at $4^{\circ} \mathrm{C}$. After washing three times with PBS, PBS was removed, organoids were resuspended in 50\% sucrose (Sigma \#84097) in PBS and stored at $4^{\circ} \mathrm{C}$ overnight. The next day, organoids were embedded in gelatin solution (7.5\% gelatin from porcine skin (Millipore \#48722) and $10 \%$ sucrose in PBS) overnight at $4^{\circ} \mathrm{C}$. The next day, organoids were mounted with Q Path Tissue OCT Medium (VWR \#0011243) to generate

30 frozen blocks which were cut into 10-14 $\mu \mathrm{m}$ sections using a Leica CM3050S cryostat. The sections were washed with PBS for 10 mins at room temperature (RT). When using 
1 biotinylated primary antibodies, slides were incubated with blocking/permeabilization buffer

2 (1\% BSA and $0.2 \%$ Triton X-100 in PBS) for 15 mins, then with blocking/permeabilization buffer

3 containing four drops per $\mathrm{ml}$ of Streptavidin Block solution (Streptavidin/Biotin Blocking Kit;

4 Vectorlabs \#SP-2002) for 15 mins, and then with blocking/permeabilization buffer containing

5 four drops per $\mathrm{ml}$ of Biotin Block solution for 15 mins. When using un-biotinylated primary

6 antibodies, slides were instead incubated with blocking/permeabilization buffer for 30 mins

7 at RT. After a quick wash in PBS slides were incubated with primary antibodies in $1 \%$ BSA in

8 PBS for $1 \mathrm{~h}$ at RT. Afterwards, slides were washed twice with PBS for each 10 mins, incubated

9 with secondary antibodies and Hoechst 33342 diluted into 1\% BSA in PBS for $1 \mathrm{~h}$ at RT, and

10 after two additional washes with PBS for 10 mins each, mounted in ProLong ${ }^{\mathrm{TM}}$ Diamond

11 Antifade Mountant (Thermo Fisher \#P36970).

12 Antibodies were: CDH1 (BD Biosciences \#610181, 1:200); EPCAM-AF647 (Abcam \#ab239273,

13 1:200); Hoechst 33342 (Thermo Fisher \#H3570; 1:10000); HOXD11 (Sigma \#SAB1403944;

14 1:300); IGF2 (Thermo Fisher \#MA5-17096; 1:200); Ki-67 (SolA15, eBisoscience \#14-5698-82,

15 1:200) (Ki-67 / Ki-67-FITC (SolA15, eBisoscience \#14-5698-82, 1:200); LHX1 (OriGene

16 \#TA504528; 1:1000); LTL-Biotinylated (Vectorlabs B-1325, 1:500); LIN28A (Cell Signaling

17 \#3978, 1:600); LIN28B (Cell Signaling \#4196, 1:250); NPHS1 (R\&D Systems \#AF4269, 1:60);

18 PAX2 (Invitrogen \#71-6000; 1:100); PAX8 (Proteintech \#10336-1-AP; 1:100); PODXL (R\&D

19 Systems \#AF1658, 1:500); SIX1 (Cell Signaling \#12891; 1:200), SIX2 (Proteintech \#11562-1-

20 AP, 1:100); WT1 (Abcam \#ab89901; 1:200); donkey-anti-rabbit AF 488 (Thermo Fisher \#A-

21 21206, 1:500); donkey-anti-rabbit AF 594 (Thermo Fisher \#A-21207, 1:500); donkey-antirabbit AF 647 (Thermo Fisher \#A-31573, 1:500); donkey-anti-goat AF 594 (Thermo Fisher \#A11058, 1:500); donkey-anti-goat AF 647 (Thermo Fisher \#A-21447, 1:500); donkey-antisheep AF 488 (Thermo Fisher \# A-11015, 1:500); donkey-anti-mouse AF 488 (Thermo Fisher \#A-21202, 1:500); donkey-anti-mouse AF 594 (Thermo Fisher \#A-21203, 1:500); donkeyanti-mouse AF 647 (Thermo Fisher \#A-31571, 1:500); donkey-anti-rat AF 647 (Abcam \#150155, 1:500); Streptavidin Fluorescent Dye 633-I (Abnova \#U0295, 1:500). Images were acquired on a Zeiss LSM710 scanning head confocal microscope and handled with Fiji software. 


\section{Bioinformatics}

2 RNA isolation of three independent biological replicates was performed as described above 3 and RNA-seq libraries prepared using the TruSeq mRNA Library preparation kit (Illumina \#20020595). RNA sequencing was performed on an Illumina HiSeq2500 machine (50 bp single-end reads). RNA-seq reads were aligned to the human hg38 genome using $q$ Align from the Bioconductor package QuasR (Gaidatzis et al, 2015) with default parameters except for aligner="Rhisat2" and splicedAlignment=TRUE. Alignments were quantified for known UCSC genes obtained from the TxDb.Hsapiens.UCSC.hg38.knownGene package using qCount (Table S1).

10 Principle component analysis (PCA) (Figure S4A) using normalized read counts of merged 11 replicates and considering the top $30 \%$ variable genes was performed using the prcomp 12 function in $\mathrm{R}$.

13 Differential gene expression was determined using edgeR (Robinson \& Oshlack, 2010). For 14 heatmap visualization (Figure 2A), 7'626 genes were considered that were significantly 15 regulated during control organoid formation (absolute log2 fold gene expression change $16(\log 2 \mathrm{FC})$ at $d 5, d 7, d 9, d 11, d 12$ or $d 21$ relative to iPSCs greater than $\log 2(3)$ with a false discovery rate (FDR) smaller than 0.001 ) or upon WT1 KO (absolute log2FC in KO ${ }^{\mathrm{iPSC}}$ or KO $\mathrm{KO}^{\mathrm{d4}-7}$ organoids relative to control organoids at any time-point greater than $\log 2(3)$ with a FDR smaller than 0.001).

For comparison with published kidney organoid gene expression (Wu et al, 2018) (GSE118184) (Figure S1C), scRNAseq reads of 218 iPSCs and of 25120 (Morizane protocol) and 82024 (Takasato protocol) cells from d26 organoids were normalized and collapsed, and $\log 2 \mathrm{FCs}$ relative to iPSCs were calculated using a pseudocount of 1 . Correlation coefficients are based on 12817 genes detected in (Wu et al, 2018) and the RNA-seq dataset reported in this work. Pearson correlation coefficients were calculated using R's cor function For generation of gene-set overlap scores (Figure 2C), we first calculated the odds-ratio for each pairwise comparison between embryonic kidney gene sets (Lindström et al, 2018) and gene clusters 1-17, using the fisher.test function in R. To correct for biases introduced by different gene-set sizes, each observed odd-ratio was then normalized by calculating a Z-score: z_score = (obs - mean_rand) / sd_rand, where obs is the observed odds-ratio for a 
1 given pairwise comparison, and mean_rand and sd_rand are the mean and standard-

2 deviation of 100 randomized odd-ratios, obtained from equal-sized sets of randomly selected

3 genes.

4 Analyses of enriched gene sets (Figure S3D and Table S1) was performed using DAVID (Huang

5 et al, 2008) and selecting GOTERM_BP_ALL.

6 Single-cell RNAseq (scRNAseq) datasets of week 16 fetal kidney scRNAseq (Hochane et al,

7 2019) (GSE114530) were integrated with and contrasted to the results of this study. For the

8 analysis and visualization of the scRNAseq data (Figures 2D) we filtered out genes detected in

$9<1 \%$ of the cells as well as the abundantly expressed and noisy ribosomal protein genes. From

10 the remaining fraction, only the top $5 \%$ overdispersed genes were selected as input for the

11 downstream dimensionality reduction and dataset integration methods according to a mean-

12 variance trend fit using a semi-parametric approach (Zheng et al, 2017). The coordinates of

13 the first 32-principal components were used to obtain the 2D tSNE representation of the data.

14 For visualization purposes per-cell gene expression values were subjected to k-nearest

15 neighbor smoothing $(k=64)$ and normalized against a random set of 2000 detected genes in

16 order to control for artefactual expression gradients.

17 For transcriptional changes in kidney cancer patients (Figure 3, S3E,F), Wilms tumor (WT;

18 Gadd et al., 2017), Kidney Chromophobe Carcinoma (KIRC; TGCA; Davis et al., 2014), Kidney

19 Papillary Cell Carcinoma (KIRP; The Cancer Genome Atlas Research Network, 2016), and

20 Kidney Clear Cell Carcinoma (KIRC; TCGA; Creighton et al., 2013) datasets (TARGET-WT, TCGA-

$21 \mathrm{KICH}$, TCGA-KIRP, and TCGA-KIRC) were downloaded from GDC

22 (https://portal.gdc.cancer.gov) using the TCGAbiolinks package available from Bioconductor.

23 Data sets were normalized, and log2FCs for each sample calculated over the mean of the

24 respective control samples using a pseudocount of 1. Correlation coefficients are for the 7'626

25 genes defined in Fig. $3 \mathrm{~A}$, and the mean of $\log 2 \mathrm{FCs}$ in $\mathrm{KO}^{\mathrm{iPSC}}$ and $\mathrm{KO}^{\mathrm{d} 4-7} \mathrm{~d} 21$ organoids.

26 Unsupervised clustering was performed using the Heatmap function from the Bioconductor

27 package ComplexHeatmap. 


\section{DATA AVAILABILITY}

2 The RNA-seq data reported in this study have been deposited at ArrayExpress with the

3 following accession number: E-MTAB-9957, and can be accessed with the Username: Reviewer_E-MTAB-9957, and the Password: Jrpdwjbn.

\section{REFERENCES}

Barak H, Huh S-H, Chen S, Jeanpierre C, Martinovic J, Parisot M, Bole-Feysot C, Nitschké $\mathrm{P}$, Salomon R, Antignac C, et al (2012) FGF9 and FGF20 Maintain the Stemness of Nephron Progenitors in Mice and Man. Dev Cell 22: 1191-1207

Ben-David U, Beroukhim R \& Golub TR (2019) Genomic evolution of cancer models: perils and opportunities. Nat Rev Cancer 19: 97-109

Berry RL, Ozdemir DD, Aronow B, Lindström NO, Dudnakova T, Thornburn A, Perry P, Baldock R, Armit C, Joshi A, et al (2015) Deducing the stage of origin of Wilms' tumours from a developmental series of Wt1-mutant mice. Dis Model Mech 8: 903-917

Bharathavikru R, Slight J, Aitken S, Petrovich G, Charlton J, Stancheva V, Essafi A, PritchardJones K \& Hastie ND (2019) Tumour suppressor WT1 regulates the let-7-Igf1r axis in kidney mesenchyme. Biorxiv: 822973

Bian S, Repic M, Guo Z, Kavirayani A, Burkard T, Bagley JA, Krauditsch C \& Knoblich JA (2018) Genetically engineered cerebral organoids model brain tumor formation. Nat Methods 15: 631-639

Calandrini C, Schutgens F, Oka R, Margaritis T, Candelli T, Mathijsen L, Ammerlaan C, Ineveld RL van, Derakhshan S, Haan S de, et al (2020) An organoid biobank for childhood kidney cancers that captures disease and tissue heterogeneity. Nat Commun 11: 1310

Chal J \& Pourquié O (2017) Making muscle: skeletal myogenesis in vivo and in vitro. Development 144: 2104-2122

Clevers H \& Tuveson DA (2019) Organoid Models for Cancer Research. Annu Rev Cancer Biology 3: 223-234

Creighton CJ, Morgan M, Gunaratne PH, Wheeler DA, Gibbs RA, Robertson AG, Chu A, Beroukhim R, Cibulskis K, Signoretti S, et al (2013) Comprehensive molecular characterization of clear cell renal cell carcinoma. Nature 499: 43-49

Davis CF, Ricketts CJ, Wang M, Yang L, Cherniack AD, Shen H, Buhay C, Kang H, Kim SC, Fahey CC, et al (2014) The Somatic Genomic Landscape of Chromophobe Renal Cell Carcinoma. Cancer Cell 26: 319-330 
Drost J, Jaarsveld RH van, Ponsioen B, Zimberlin C, Boxtel R van, Buijs A, Sachs N, Overmeer RM, Offerhaus GJ, Begthel $H$, et al (2015) Sequential cancer mutations in cultured human intestinal stem cells. Nature 521: 43-47

Essafi A, Webb A, Berry RL, Slight J, Burn SF, Spraggon L, Velecela V, Martinez-Estrada OM, Wiltshire JH, Roberts SGE, et al (2011) A Wt1-Controlled Chromatin Switching Mechanism Underpins Tissue-Specific Wnt4 Activation and Repression. Dev Cell 21: 559574

Fessler E, Drost J, Hooff SR, Linnekamp JF, Wang X, Jansen M, Melo FDSE, Prasetyanti PR, IJspeert JE, Franitza M, et al (2016) TGF $\beta$ signaling directs serrated adenomas to the mesenchymal colorectal cancer subtype. Embo Mol Med 8: 745-760

Fumagalli A, Drost J, Suijkerbuijk SJE, Boxtel R van, Ligt J de, Offerhaus GJ, Begthel H, Beerling E, Tan EH, Sansom OJ, et al (2017) Genetic dissection of colorectal cancer progression by orthotopic transplantation of engineered cancer organoids. Proc National Acad Sci 114: E2357-E2364

Gadd S, Huff V, Huang C-C, Ruteshouser EC, Dome JS, Grundy PE, Breslow N, Jennings L, Green DM, Beckwith JB, et al (2012) Clinically Relevant Subsets Identified by Gene Expression Patterns Support a Revised Ontogenic Model of Wilms Tumor: A Children's Oncology Group Study. Neoplasia 14: 742-IN21

Gadd S, Huff V, Walz AL, Ooms AHAG, Armstrong AE, Gerhard DS, Smith MA, Auvil JMG, Meerzaman D, Chen Q-R, et al (2017) A Children's Oncology Group and TARGET initiative exploring the genetic landscape of Wilms tumor. Nat Genet 49: 1487-1494

Gaidatzis D, Lerch A, Hahne F \& Stadler MB (2015) QuasR: quantification and annotation of short reads in R. Bioinformatics 31: 1130-1132

Hanahan D \& Coussens LM (2012) Accessories to the Crime: Functions of Cells Recruited to the Tumor Microenvironment. Cancer Cell 21: 309-322

Hastie ND (2017) Wilms' tumour 1 (WT1) in development, homeostasis and disease. Development 144: 2862-2872

Hochane M, Berg PR van den, Fan X, Bérenger-Currias N, Adegeest E, Bialecka M, Nieveen M, Menschaart M, Lopes SMC de S \& Semrau S (2019) Single-cell transcriptomics reveals gene expression dynamics of human fetal kidney development. Plos Biol 17: e3000152

Huang DW, Sherman BT \& Lempicki RA (2008) Bioinformatics enrichment tools: paths toward the comprehensive functional analysis of large gene lists. Nucleic Acids Res 37: 113

Huang L, Mokkapati S, Hu Q, Ruteshouser EC, Hicks MJ \& Huff V (2016) Nephron Progenitor But Not Stromal Progenitor Cells Give Rise to Wilms Tumors in Mouse Models with $\beta$ Catenin Activation or Wt1 Ablation and Igf2 Upregulation. Neoplasia 18: 71-81 
Hynds RE, Vladimirou E \& Janes SamM (2018) The secret lives of cancer cell lines. Dis Model Mech 11: dmm037366

Kann M, Ettou S, Jung YL, Lenz MO, Taglienti ME, Park PJ, Schermer B, Benzing T \& Kreidberg JA (2015) Genome-Wide Analysis of Wilms' Tumor 1-Controlled Gene Expression in Podocytes Reveals Key Regulatory Mechanisms. J Am Soc Nephrol 26: 2097-2104

Kawasaki K, Toshimitsu K, Matano M, Fujita M, Fujii M, Togasaki K, Ebisudani T, Shimokawa M, Takano A, Takahashi S, et al (2020) An Organoid Biobank of Neuroendocrine Neoplasms Enables Genotype-Phenotype Mapping. Cell 183: 1420-1435.e21

Kersten K, Visser KE de, Miltenburg MH van \& Jonkers J (2017) Genetically engineered mouse models in oncology research and cancer medicine. Embo Mol Med 9: 137-153

Kreidberg JA, Sariola H, Loring JM, Maeda M, Pelletier J, Housman D \& Jaenisch R (1993) WT1 is required for early kidney development. Cell 74: 679-691

Lindström NO, Brandine GDS, Tran T, Ransick A, Suh G, Guo J, Kim AD, Parvez RK, Ruffins SW, Rutledge EA, et al (2018) Progressive Recruitment of Mesenchymal Progenitors Reveals a Time-Dependent Process of Cell Fate Acquisition in Mouse and Human Nephrogenesis. Dev Cell 45: 651-660.e4

Liu Z, Lou H, Xie K, Wang H, Chen N, Aparicio OM, Zhang MQ, Jiang R \& Chen T (2017) Reconstructing cell cycle pseudo time-series via single-cell transcriptome data. Nat Commun 8: 22

Matano M, Date S, Shimokawa M, Takano A, Fujii M, Ohta Y, Watanabe T, Kanai T \& Sato T (2015) Modeling colorectal cancer using CRISPR-Cas9-mediated engineering of human intestinal organoids. Nat Med 21: 256-262

Miyagawa K, Kent J, Moore A, Charlieu J-P, Little MH, Williamson KA, Kelsey A, Brown KW, Hassam S, Briner J, et al (1998) Loss of WT1 function leads to ectopic myogenesis in Wilms' tumour. Nat Genet 18: 15-17

Morizane R, Lam AQ, Freedman BS, Kishi S, Valerius MT \& Bonventre JV (2015) Nephron organoids derived from human pluripotent stem cells model kidney development and injury. Nat Biotechnol 33: 1193-1200

Motamedi FJ, Badro DA, Clarkson M, Lecca MR, Bradford ST, Buske FA, Saar K, Hübner N, Brändli AW \& Schedl A (2014) WT1 controls antagonistic FGF and BMP-pSMAD pathways in early renal progenitors. Nat Commun 5: 4444

Network CGAR, Linehan WM, Spellman PT, Ricketts CJ, Creighton CJ, Fei SS, Davis C, Wheeler DA, Murray BA, Schmidt L, et al (2016) Comprehensive Molecular Characterization of Papillary Renal-Cell Carcinoma. New Engl J Medicine 374: 135-145

Ogawa J, Pao GM, Shokhirev MN \& Verma IM (2018) Glioblastoma Model Using Human Cerebral Organoids. Cell Reports 23: 1220-1229 
Pode-Shakked N, Gershon R, Tam G, Omer D, Gnatek Y, Kanter I, Oriel S, Katz G, HarariSteinberg O, Kalisky T, et al (2017) Evidence of In Vitro Preservation of Human Nephrogenesis at the Single-Cell Level. Stem Cell Rep 9: 279-291

Poladia DP, Kish K, Kutay B, Hains D, Kegg H, Zhao H \& Bates CM (2006) Role of fibroblast growth factor receptors 1 and 2 in the metanephric mesenchyme. Dev Biol 291: 325-339

Robinson MD \& Oshlack A (2010) A scaling normalization method for differential expression analysis of RNA-seq data. Genome Biol 11: R25

Sampson VB, David JM, Puig I, Patil PU, Herreros AG de, Thomas GV \& Rajasekaran AK (2014) Wilms' Tumor Protein Induces an Epithelial-Mesenchymal Hybrid Differentiation State in Clear Cell Renal Cell Carcinoma. Plos One 9: e102041

Schumacher V, Schneider S, Figge A, Wildhardt G, Harms D, Schmidt D, Weirich A, Ludwig R \& Royer-Pokora B (1997) Correlation of germ-line mutations and two-hit inactivation of the WT1 gene with Wilms tumors of stromal-predominant histology. Proc National Acad Sci 94: 3972-3977

Shukrun R, Pode-Shakked N, Pleniceanu O, Omer D, Vax E, Peer E, Pri-Chen S, Jacob J, Hu Q, Harari-Steinberg O, et al (2014) Wilms' Tumor Blastemal Stem Cells Dedifferentiate to Propagate the Tumor Bulk. Stem Cell Rep 3: 24-33

Treger TD, Chowdhury T, Pritchard-Jones K \& Behjati S (2019) The genetic changes of Wilms tumour. Nat Rev Nephrol 15: 240-251

Villegas F, Lehalle D, Mayer D, Rittirsch M, Stadler MB, Zinner M, Olivieri D, Vabres $P$, Duplomb-Jego L, Bont ESJMD, et al (2019) Lysosomal Signaling Licenses Embryonic Stem Cell Differentiation via Inactivation of Tfe3. Cell Stem Cell 24: 257-270.e8

Walz AL, Ooms A, Gadd S, Gerhard DS, Smith MA, Auvil JMG, Auvil JMG, Meerzaman D, Chen Q-R, Hsu CH, et al (2015) Recurrent DGCR8, DROSHA, and SIX Homeodomain Mutations in Favorable Histology Wilms Tumors. Cancer Cell 27: 286-297

Wegert J, Ishaque N, Vardapour R, Geörg C, Gu Z, Bieg M, Ziegler B, Bausenwein S, Nourkami $\mathrm{N}$, Ludwig N, et al (2015) Mutations in the SIX1/2 Pathway and the DROSHA/DGCR8 miRNA Microprocessor Complex Underlie High-Risk Blastemal Type Wilms Tumors. Cancer Cell 27: 298-311

Wegert J, Zauter L, Appenzeller S, Otto C, Bausenwein S, Vokuhl C, Ernestus K, Furtwängler R, Graf N \& Gessler M (2020) High-risk blastemal Wilms tumor can be modeled by 3D spheroid cultures in vitro. Oncogene 39: 849-861

Wu H, Uchimura K, Donnelly EL, Kirita Y, Morris SA \& Humphreys BD (2018) Comparative Analysis and Refinement of Human PSC-Derived Kidney Organoid Differentiation with Single-Cell Transcriptomics. Cell Stem Cell 23: 869-881.e8 
1 Zheng GXY, Terry JM, Belgrader P, Ryvkin P, Bent ZW, Wilson R, Ziraldo SB, Wheeler TD, 2 McDermott GP, Zhu J, et al (2017) Massively parallel digital transcriptional profiling of $3 \quad$ single cells. Nat Commun 8: 14049 
A

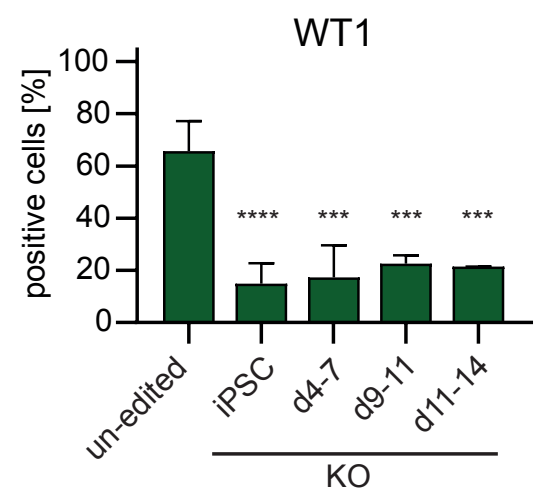

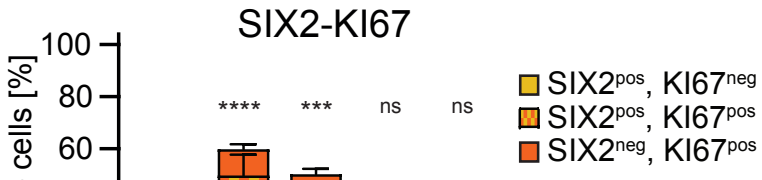

B

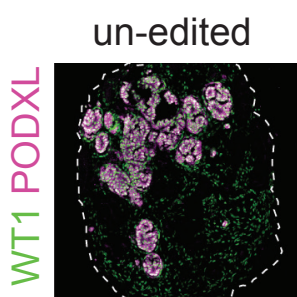

$\mathrm{KO}^{\mathrm{iPSC}}$

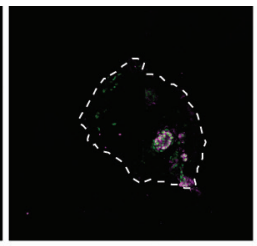

$\mathrm{KO}^{\mathrm{d} 4-7}$

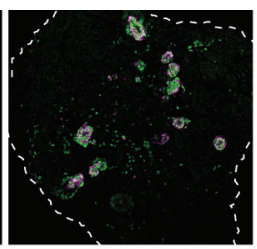

$\mathrm{KO} 9 \mathrm{~d}-11$

$\mathrm{KO}^{\mathrm{d} 11-14}$
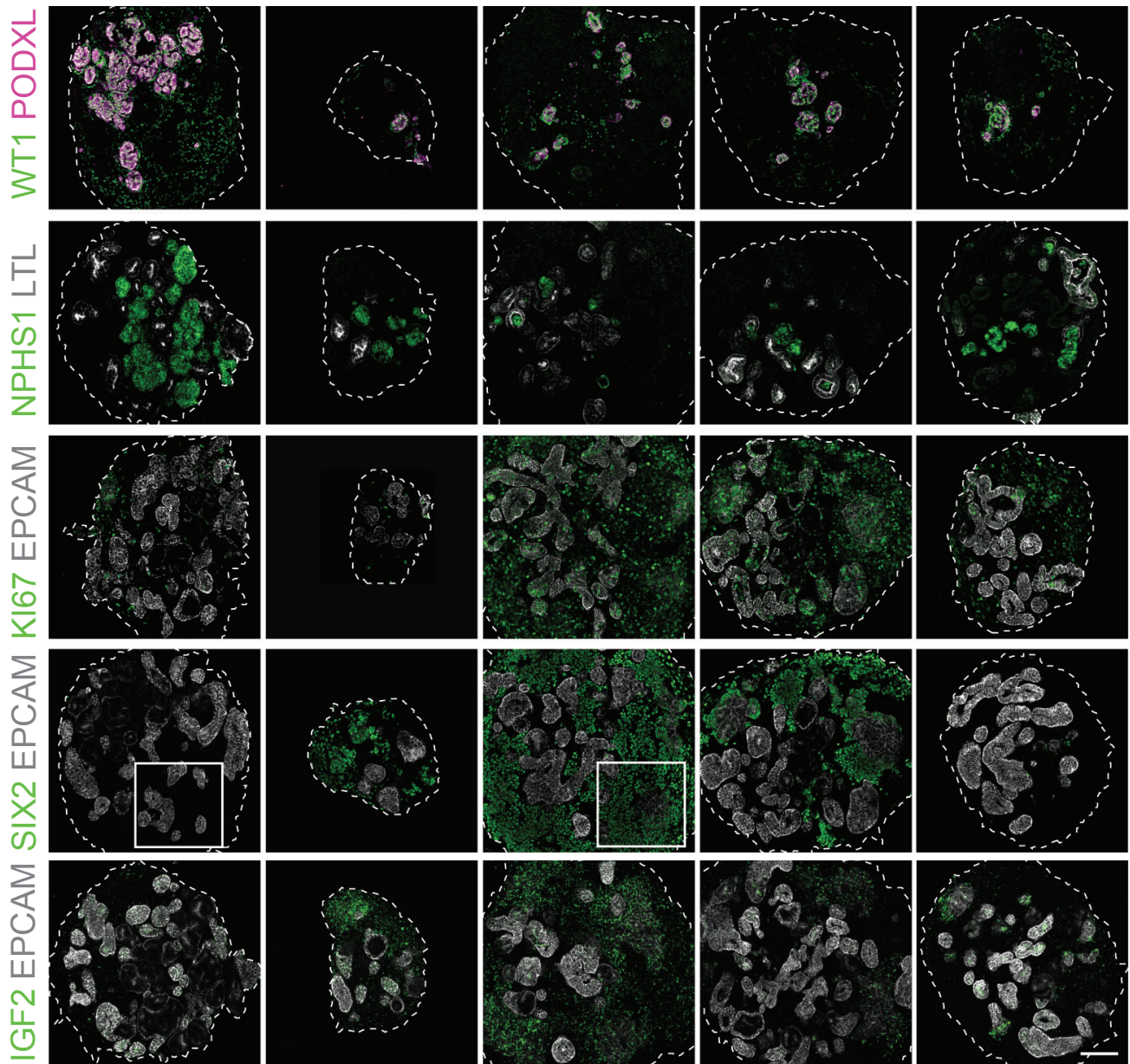

C

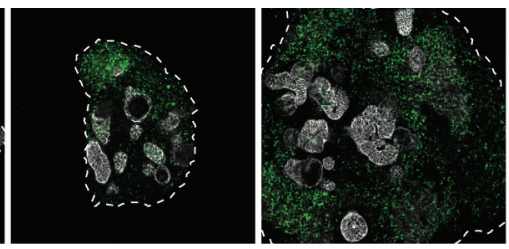

Day 10

Day 21
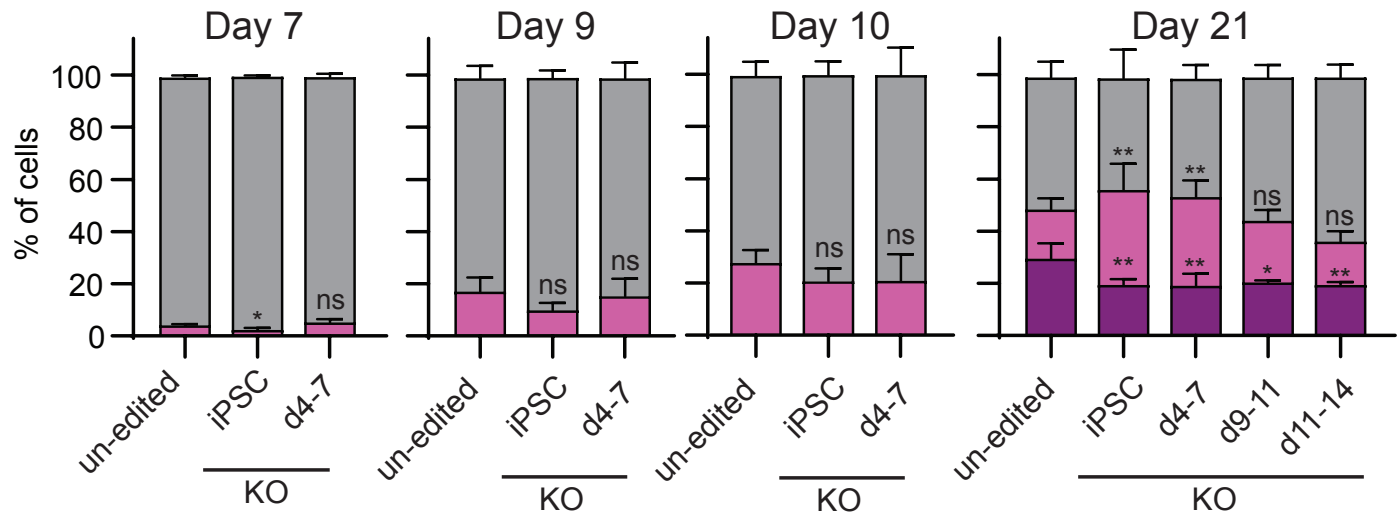

$\square$ EPCAM $^{\text {low }}$

$\square$ EPCAM $^{\text {mid }}$

$\square$ EPCAM ${ }^{\text {high }}$ 
bioRxiv preprint doi: https://doi.org/10.1101/2021.02 02 429313; this version posted February 2, 2021. The copyright holder for this Waehle etearit., (ntifbyafetertified by peer review) is the author/funder. All rights reserved. No reuse allowed without permission.

A

$\Delta$ time

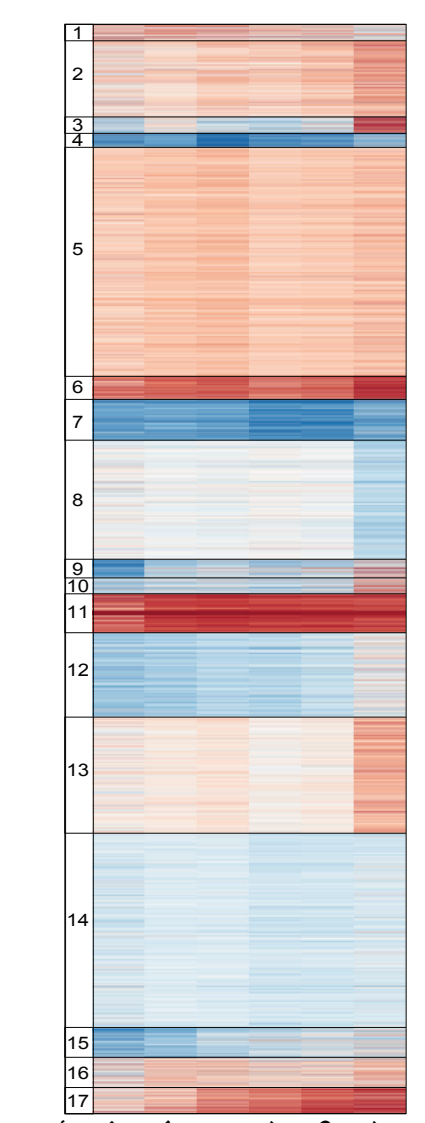

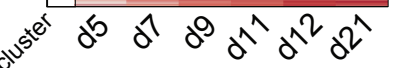

C

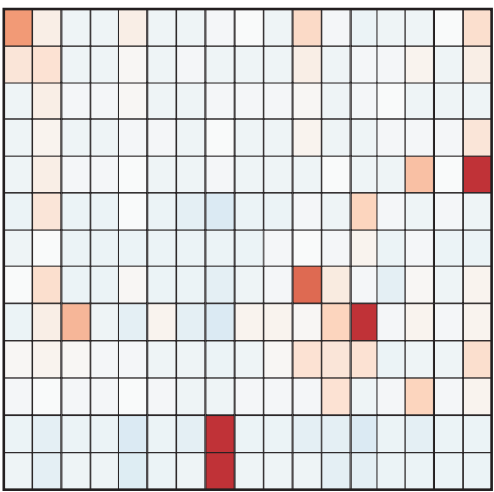

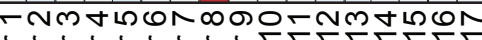

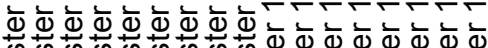

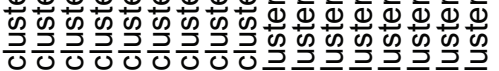
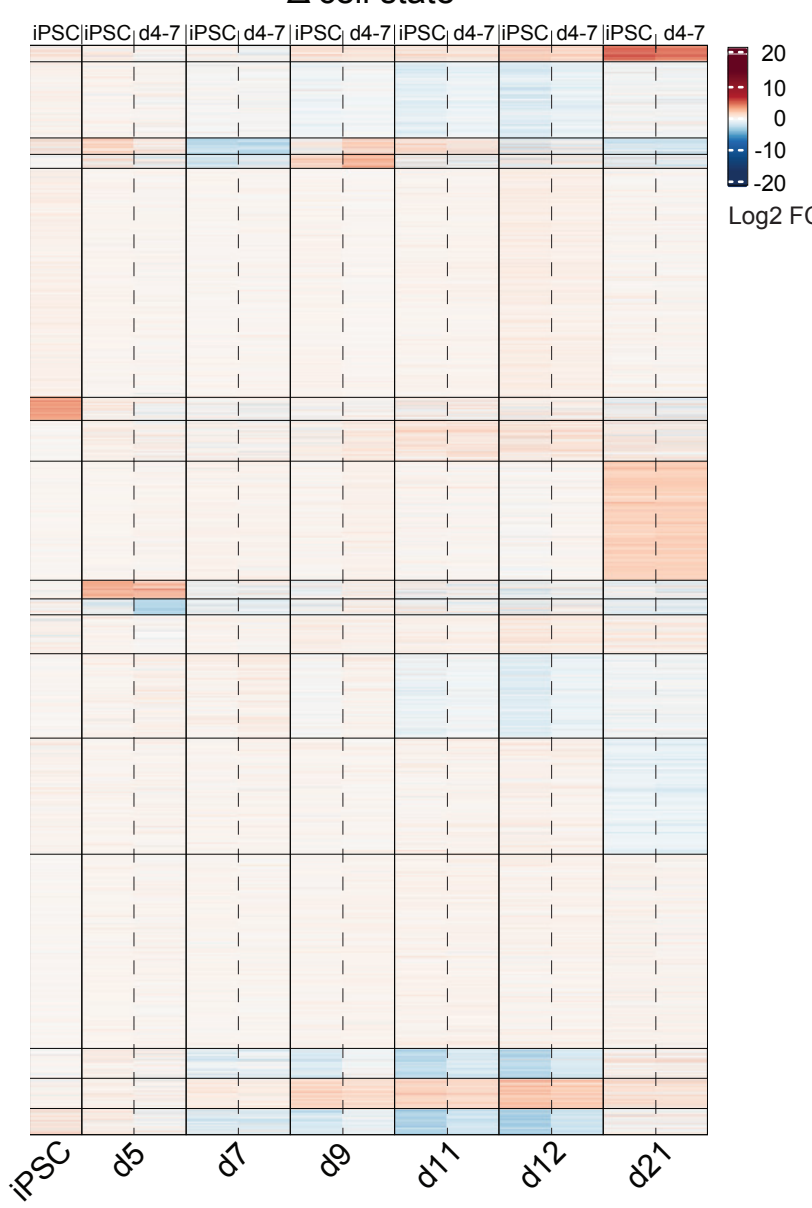

D

M1 NPCs

M2 NPCs primed to diff. M3 Differentiating NPCs

M4 Differentiation I

M5 Differentiation II

M6 Podocyte

M7 Medial/Proximal

M8 Medial/Distal

M9 Proximal tubule

M10 Loop of Henle

M11 Connecting tubule

M12 Kidney proliferation I

M13 Kidney proliferation II

60
40
20
0
-20

Z score
B
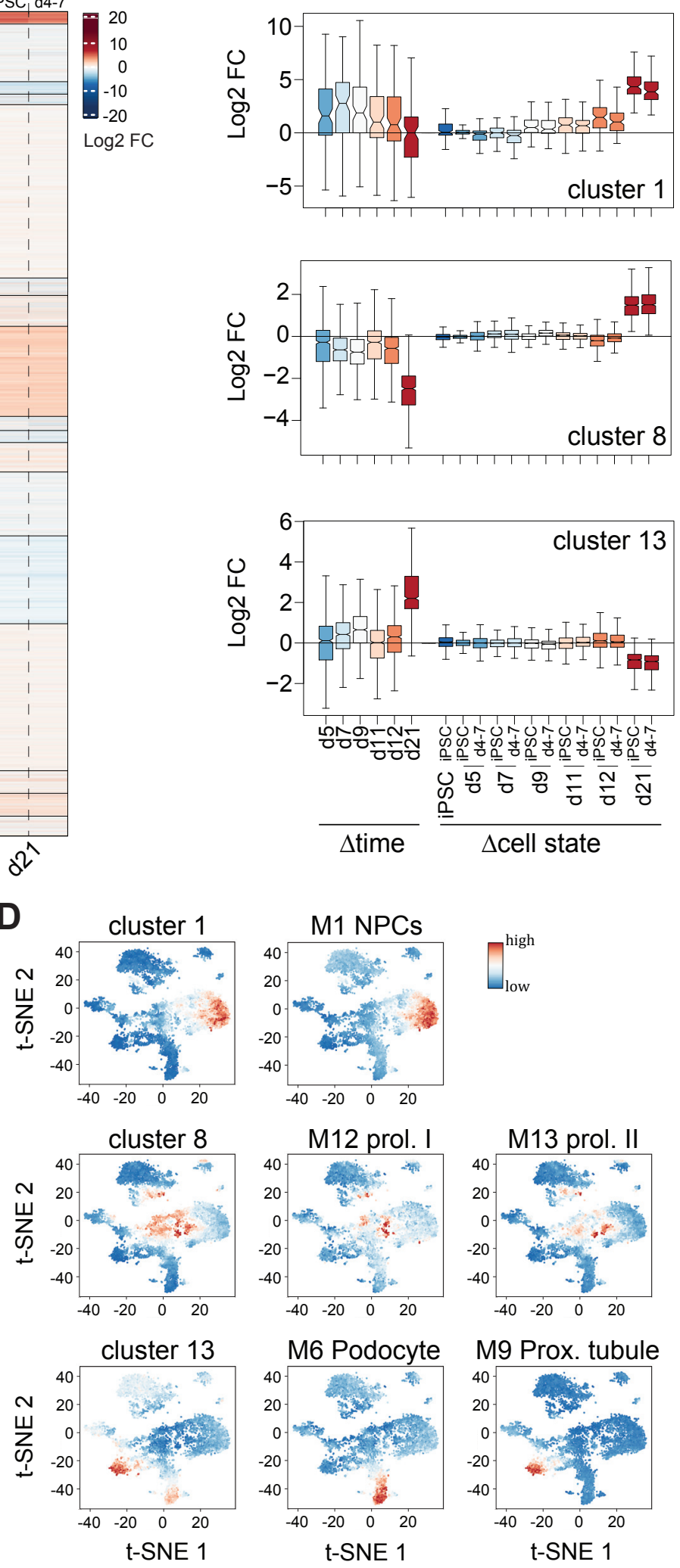

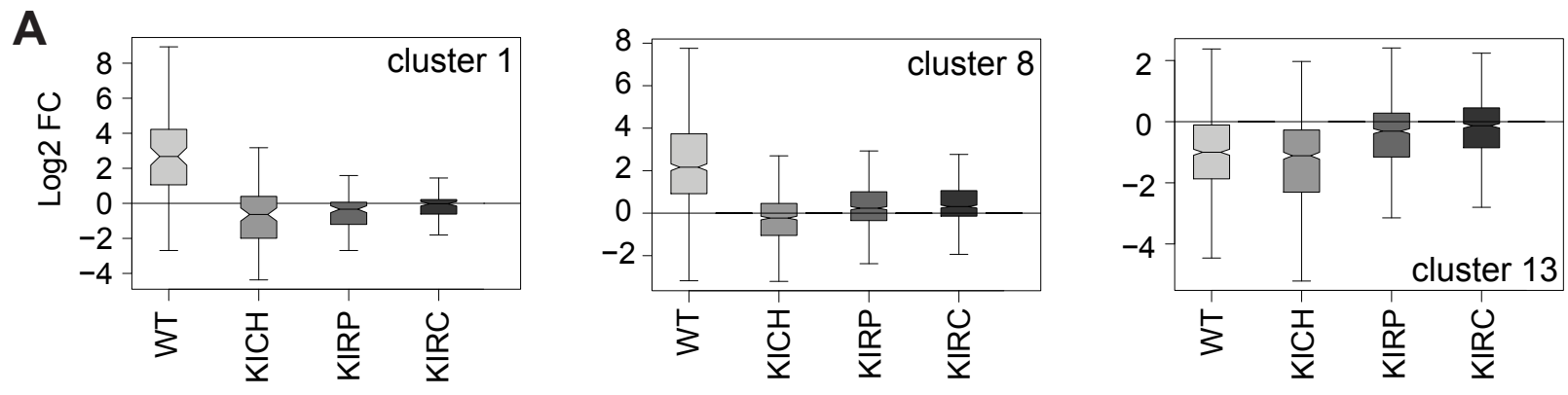

B

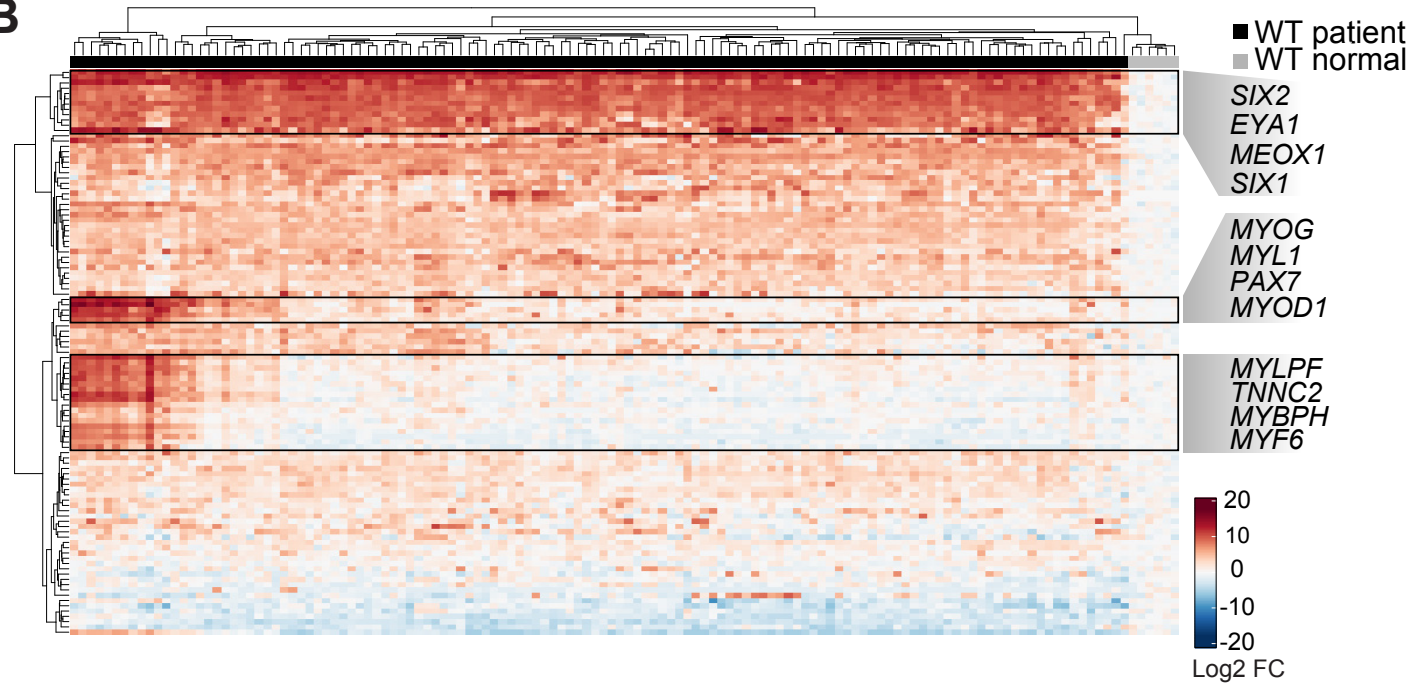


A

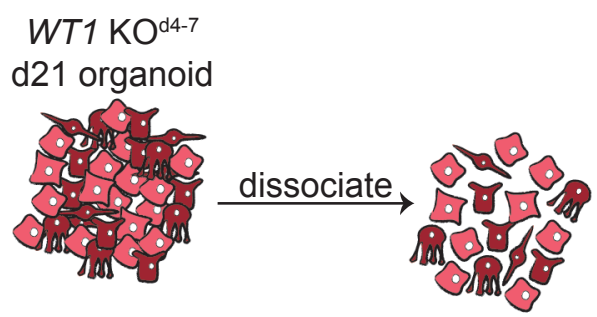

wildtype GFP pos d9 NPCs passage

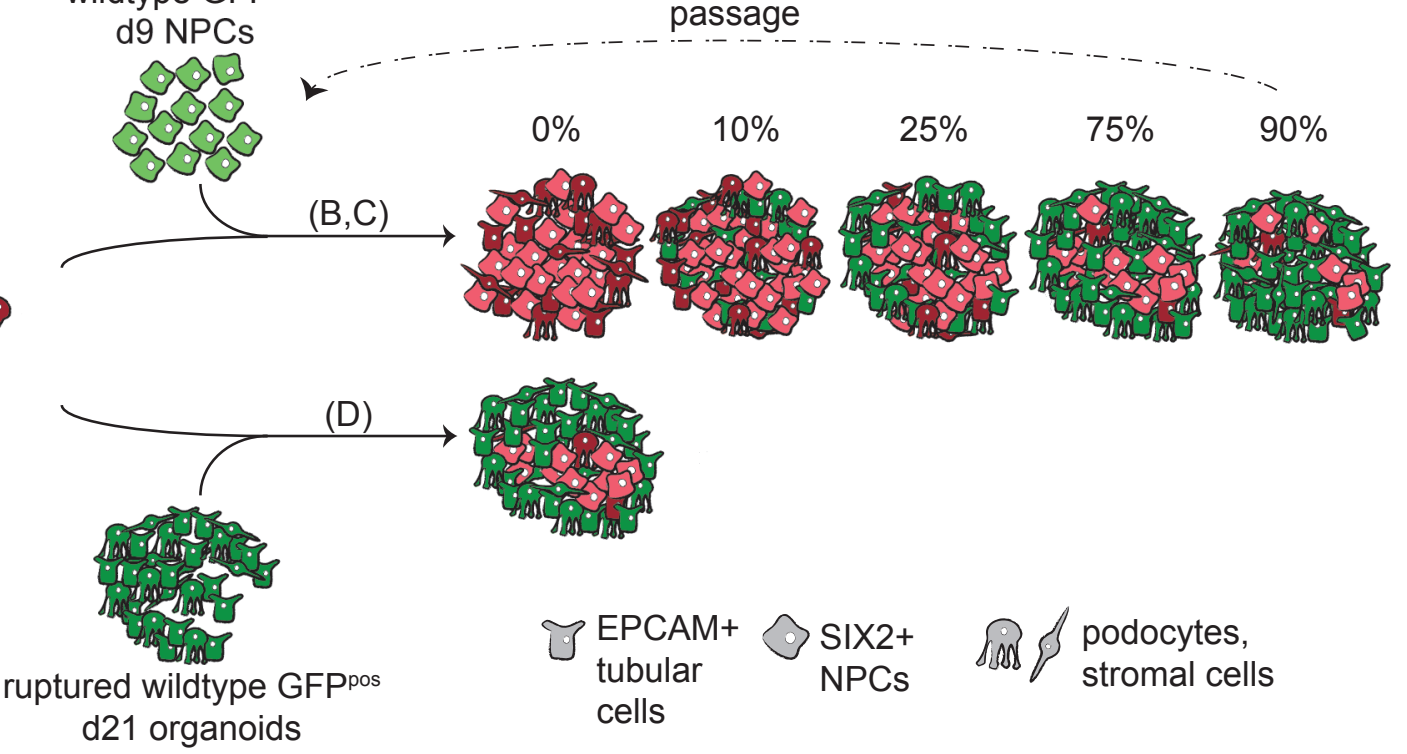

B
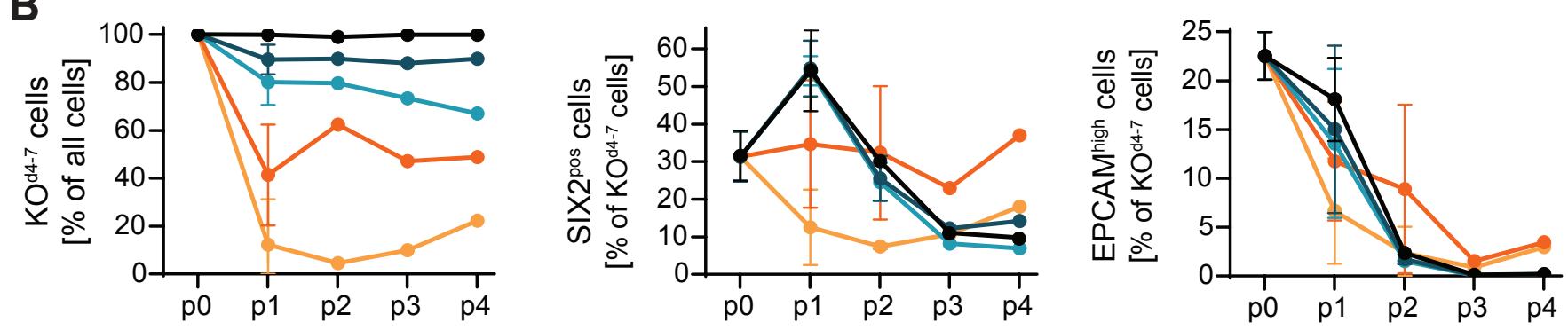

C
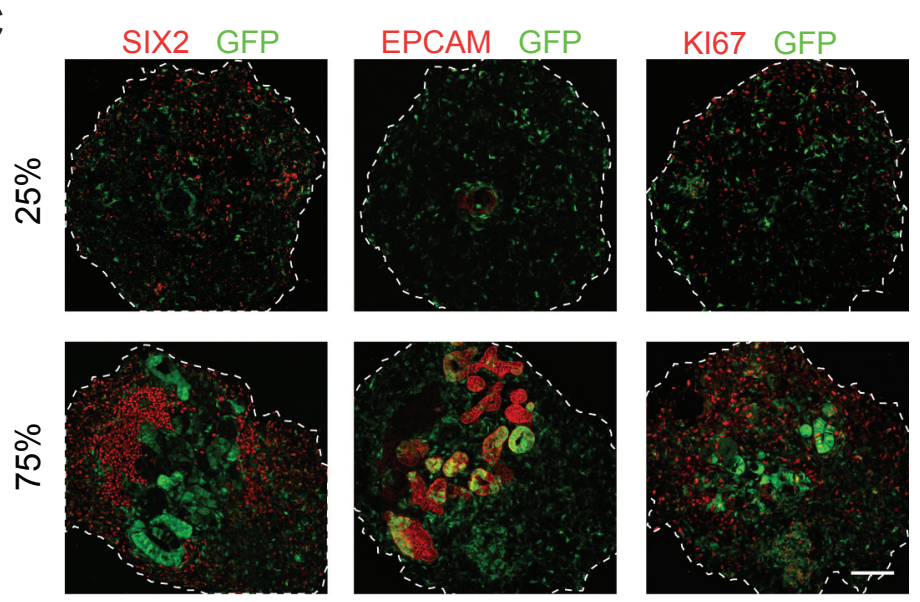

D

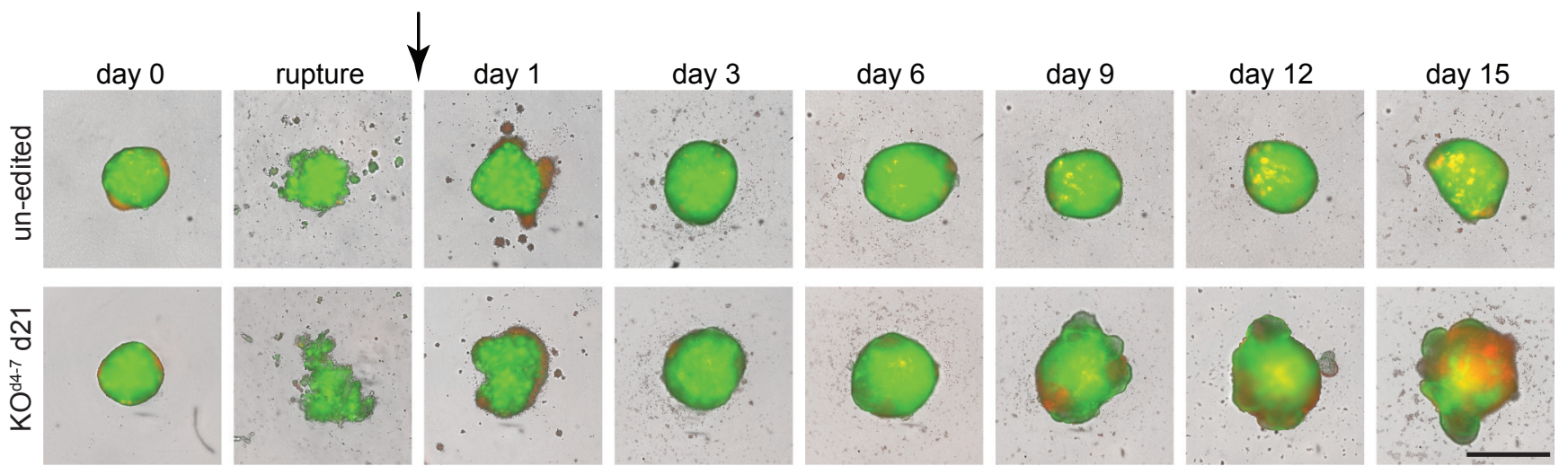

